\title{
Self-interactions of the lightest minimal supersymmetric standard model Higgs boson in the large pseudoscalar-mass limit
}

\author{
A. Dobado, ${ }^{1, *}$ M. J. Herrero, ${ }^{2, \dagger}$ W. Hollik, ${ }^{3, \ddagger}$ and S. Peñaranda ${ }^{4,8}$ \\ ${ }^{1}$ Departamento de Física Teórica, Universidad Complutense de Madrid, 28040 Madrid, Spain \\ ${ }^{2}$ Departamento de Física Teórica, Universidad Autónoma de Madrid, Cantoblanco, 28049 Madrid, Spain \\ ${ }^{3}$ Max-Planck-Institut für Physik, Föhringer Ring 6, D-80805 München, Germany \\ ${ }^{4}$ Institut für Theoretische Physik, Universität Karlsruhe Kaiserstraße 12, D-76128 Karlsruhe, Germany
}

(Received 2 September 2002; published 27 November 2002)

\begin{abstract}
We investigate the decoupling properties of the Higgs-sector-induced one-loop corrections in the lightest Higgs-boson self-couplings, in the framework of the minimal supersymmetric standard model (MSSM). The renormalized $n$-point vertex functions with external Higgs particles in the MSSM and in the SM are derived to the one-loop level and compared in the $M_{A^{0}} \gg M_{Z}$ limit. The computation has been done in a general $R_{\xi}$ gauge and the on-shell renormalization scheme is chosen. By a comparison of the renormalized lightest Higgs-boson $h^{0}$ vertex functions with respect to the corresponding SM ones, we find that the differences between the predictions of both models are summarized in the lightest Higgs-boson mass correction $\Delta M_{h^{0}}$. Consequently, the radiative corrections are absorbed in the Higgs-boson mass, and the trilinear and quartic $h^{0}$ self-couplings acquire the same structure as the couplings of the SM Higgs boson. Therefore, decoupling of the heavy MSSM Higgs bosons occurs and the MSSM $h^{0}$ self-interactions converge to the SM ones in the $M_{A^{0}} \gg M_{Z}$ limit.
\end{abstract}

DOI: 10.1103/PhysRevD.66.095016

PACS number(s): 12.60.Jv, 12.15.Lk, 14.80.Bn, 14.80.Ly

\section{INTRODUCTION}

The expectations of the discovery of at least one light Higgs particle at the next generation of high-energy colliders have greatly increased in recent years after the valuable data taken at the CERN $e^{+} e^{-}$Collider LEP and Fermilab Tevatron [1]. The standard model (SM) Higgs-boson mass $M_{H_{S M}}$ is now constrained by the worldwide electroweak data to be $M_{H_{S M}}<195 \mathrm{GeV}$ and by the direct search performed at the LEP II machine to be $M_{H_{S M}}>114.1 \mathrm{GeV}$, both at $95 \%$ C.L. In the minimal supersymmetric standard model (MSSM), on the other hand, the mass of the lightest neutral Higgs particle, $M_{h^{0}}$, is predicted to be bounded from above by $M_{h^{0}}$ $\lesssim 135 \mathrm{GeV}$ and the direct searches at LEP give a 95\% C.L. exclusion limit of $M_{h^{0}}>91 \mathrm{GeV}$. This remarkable shrinkage of the allowed mass range of these Higgs particles has enhanced even more the expectations for their discovery at the forthcoming CERN Large Hadron Collider (LHC) and the next runs of the Fermilab Tevatron.

Assuming the hypothetical discovery of one of these two light Higgs particles in the next generation of colliders, the next challenge will be to measure its mass and couplings to all known particles, including its couplings to SM fermions and SM gauge bosons, as well as the Higgs-particle selfcouplings themselves. The measurement of these parameters can serve to unravel the supersymmetric (SUSY) or nonsupersymmetric origin of this Higgs particle, and, more specifically, to distinguish if this is the SM, $H_{\mathrm{SM}}$, or the MSSM, $h^{0}$. Particularly relevant will be the measurement of the Higgs-boson self-couplings in order to establish the Higgs

\footnotetext{
*Email address: malcon@fis.ucm.es

${ }^{\dagger}$ Email address: herrero@delta.ft.uam.es

*Email address: hollik@mppmu.mpg.de

${ }^{\S}$ Email address: siannah@particle.uni-karlsruhe.de
}

mechanism experimentally. The reconstruction of the needed self-interaction potential requires a knowledge of both the trilinear and quartic self-couplings of the Higgs boson. Since the predictions of these self-couplings are different in the SM and in the MSSM, their experimental measurement could provide not just an essential way to determine the mechanism for generating the masses of the fundamental particles but also an indirect way to test supersymmetry. In the SM, at the tree level, the Higgs boson self-couplings are uniquely determined by the Higgs-boson mass $M_{H_{S M}}$ and the vacuum expectation value of the Higgs-boson field $v$, or equivalently the $W$ boson mass $M_{W}$ and the $\mathrm{SU}(2)_{L}$ gauge coupling $g$, since $v=2 M_{W} / g$. More specifically, $\lambda_{H H H}=3 M_{H_{S M}}^{2} / v$ and $\lambda_{H H H H}=3 M_{H_{S M}}^{2} / v^{2}$. In contrast, in the MSSM [2], the treelevel trilinear and quartic $h^{0}$ couplings are determined by the $\mathrm{SU}(2)_{L}$ gauge coupling $g$, the weak angle $\theta_{W}$, the $Z$ boson mass $M_{Z}$, the ratio of the two Higgs-boson vacuum expectation values, $\tan \beta=v_{2} / v_{1}$, and the $C P$-odd Higgs-boson mass $M_{A^{0}}$, that is, $\lambda_{h h h}^{0}=3\left(g M_{Z} / 2 c_{W}\right) \cos 2 \alpha \sin (\beta+\alpha)$ and $\lambda_{h h h h}^{0}=3\left(g^{2} / 4 c_{W}^{2}\right) \cos ^{2} 2 \alpha$, with the mixing angle $\alpha$ and $M_{h^{0}}$ being derived quantities from $\beta, M_{A^{0}}$, and $M_{Z}$. For arbitrary values of the MSSM Higgs-sector input parameters $\tan \beta$ and $M_{A^{0}}$, the values of these self-couplings are clearly different from those of their corresponding trilinear and quartic SM couplings. However, the situation changes in the large pseudoscalar-mass limit $M_{A^{0}} \gg M_{Z}$, yielding a particular spectrum with heavy $H^{0}, H^{ \pm}, A^{0}$ Higgs bosons having similar masses $M_{A^{0}} \simeq M_{H^{ \pm}} \simeq M_{H^{0}}$, and a light $h^{0}$ boson having a

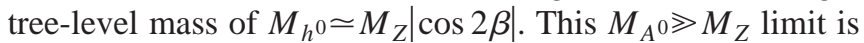
referred to in the literature (and in the present work from now on) as the decoupling limit of the MSSM Higgs sector [3], because the $h^{0}$ tree-level interactions with the SM fermions and SM gauge bosons resemble the corresponding SM Higgs boson interactions. Furthermore, in this large pseudoscalar-mass limit, which also implies $\alpha \rightarrow \beta$ 
$-\pi / 2$, the $h^{0}$ self-couplings approach, respectively, $\lambda_{h h h}^{0}$ $\simeq 3 g / 2 M_{W^{\prime}} M_{h^{0}}^{2}$ and $\lambda_{h h h h}^{0} \simeq 3 g^{2} / 4 M_{W}^{2} M_{h^{0}}^{2}$ and, therefore, they converge as well to their respective SM Higgs boson self-couplings if $M_{H_{S M}}$ is taken to be equal to $M_{h^{0}}$. We can therefore conclude that, at the tree level, there is decoupling of the heavy MSSM Higgs sector and by studying the light Higgs boson self-interactions it will be very difficult to unravel its SUSY origin.

In this paper we are concerned with the behavior of the self-interactions of the lightest MSSM Higgs boson beyond the tree level, where important radiative corrections from various sectors are expected [4-10]. In particular, the oneloop corrections from the quark and squark sectors are known to be large, specially in the low $\tan \beta$ and $M_{A^{0}}$ region where they can amount up to $5 \%$ even for heavy squarks in the $\mathrm{TeV}$ region [9]. We focus here on the one-loop radiative corrections to the $h^{0}$ self-couplings from the MSSM Higgs sector itself, and study the decoupling behavior of these corrections in the limit where $H^{0}, H^{ \pm}$, and $A^{0}$ become quasidegenerate and very heavy as compared to the electroweak scale, while $h^{0}$ remains light, $M_{h^{0}} \lesssim 135 \mathrm{GeV}$. We address the question above about the possible convergence or divergence of these self-couplings to the SM ones and draw conclusions about the important issue of the possibility of discerning between $h^{0}$ and $H_{\mathrm{SM}}$ in the decoupling limit through the study of their self-interactions.

From the more formal point of view of the effective field theory, such study corresponds to determining the lowenergy effective action describing the $h^{0}$ self-interactions that is obtained after integration of the heavy Higgs-boson fields, $H^{0}, H^{ \pm}$, and $A^{0}$, and to deciding if these effective $h^{0}$ self-interactions, which are valid at low energies $E \ll M_{A^{0}}$, are the same or not as the SM ones. In fact, whenever a symmetry is present in a fundamental theory and one is interested in having this symmetry also in low-energy effective theory, the particles to be integrated must satisfy a complete representation of that symmetry. In our case, the MSSM plays the role of the fundamental theory and it is $\mathrm{SU}(2)_{L}$ $\times \mathrm{U}(1)_{Y}$ gauge invariant. Therefore, the SM, which is also gauge invariant, could be obtained in principle as an effective theory from the MSSM only if one integrates both of the Higgs MSSM doublets which include $H^{0}, H^{ \pm}, A^{0}$, the Goldstone bosons, and the $h^{0}$ itself, and not only the heavy modes. This is why we consider here the integration of all the MSSM Higgs-boson modes.

The computation of the low-energy $h^{0}$ self-interactions can be performed in two ways: either by integrating out the Higgs-boson fields in the path integral formalism [11,12], or by standard Feynman-diagrammatic methods. We will choose this second method here and proceed as follows. We evaluate the one-particle irreducible (1PI) Green functions with external $h^{0}$ particles to one-loop level and then we evaluate the corresponding renormalized 1PI Green functions by fixing the on-shell renormalization scheme. We will concentrate on studying the behavior of these renormalized vertex functions in the decoupling limit where $H^{0}, H^{ \pm}$, and $A^{0}$ are much heavier than $Z$, while both the $h^{0}$ mass $M_{h^{0}}$ and the momenta of the external $h^{0}$ particles remain at some low-energy scale below $M_{A^{0}}$. This will give us the values of the low-energy $h^{0}$ self-couplings that we are looking for. In order to address the comparison with the $H_{\mathrm{SM}}$ self-couplings we follow the so-called matching procedure [13] in which the quantities to be compared are the renormalized 1PI Green functions of the two theories. More concretely, we compare here the renormalized $h^{0}$ 1PI Green functions, in the previously mentioned decoupling limit, and the corresponding SM renormalized $H_{\mathrm{SM}}$ 1PI functions at the one-loop level, and we find that they are indeed equal for all the studied $n$-point functions $(n=1, \ldots, 4)$. In particular, the $n=3,4$ cases show explicitly the convergence of the MSSM $h^{0}$ self-couplings to the $H_{\mathrm{SM}}$ self-couplings at the one-loop level that we are looking for. We also show that all the one-loop effects from the heavy Higgs-boson modes $H^{0}, H^{ \pm}$, and $A^{0}$ in the lowenergy $h^{0}$ self-interactions either are absorbed into a redefinition of the low-energy parameters (concretely, $M_{h^{0}}$ ), or else are suppressed by inverse powers of $M_{A^{0}}$. Consequently, and following the lines of the Appelquist-Carazzone theorem [14], we conclude that the heavy Higgs bosons $H^{0}, H^{ \pm}$, and $A^{0}$ do decouple from the low-energy $h^{0}$ self-interactions, not just at the tree level but also at one-loop level. This indicates that it will therefore be very difficult, even with highprecision experiments, to distinguish an $h^{0}$ from the SM Higgs boson by studying their self-interactions, if the pseudoscalar boson mass turns out to be large.

The paper is organized as follows. In Sec. II we briefly present those aspects of the MSSM that we are interested in, fixing our notation. A discussion of the one-loop MSSM Higgs-sector contributions and the analytical results of these contributions to the $h^{0}$ self-interactions in the decoupling limit are included in Sec. II A. Section II B is devoted to the on-shell renormalization procedure, where the expressions for the $n$-point vertex function counterterms, in the $M_{A^{0}}$ $\gg M_{Z}$ limit, and also the explicit asymptotic results for the renormalization constants are presented. Finally, in this subsection we give the renormalized vertex results in the decoupling limit. A discussion of the Higgs-boson self-couplings in the SM, by giving the one-loop $H_{\mathrm{SM}}$ self-coupling corrections, the results for the renormalization constants by assuming the on-shell scheme, and finally the SM renormalized vertex functions, is presented in Sec. III. A comparison of the results for the renormalized $n$-point functions in the two theories is examined and discussed in detail in Sec. IV. Finally, the summary of our conclusions is presented at the end of this last section.

\section{MSSM HIGGS SECTOR}

The Higgs sector of the MSSM involves two scalar doublets $H_{1}$ and $H_{2}$, in order to give masses to up- and downtype fermions in a way consistent with supersymmetry. The two-doublet Higgs potential is given by [2]

$$
\begin{aligned}
V= & m_{1}^{2} H_{1} \bar{H}_{1}+m_{2}^{2} H_{2} \bar{H}_{2}+m_{12}^{2}\left(\epsilon_{a b} H_{1}^{a} H_{2}^{b}+\text { H.c. }\right) \\
& +\frac{g^{\prime 2}+g^{2}}{8}\left(H_{1} \bar{H}_{1}-H_{2} \bar{H}_{2}\right)^{2}+\frac{g^{2}}{2}\left|H_{1} \bar{H}_{2}\right|^{2}
\end{aligned}
$$


with the doublet fields $\mathrm{H}_{1}$ and $\mathrm{H}_{2}$, the soft SUSY-breaking terms $m_{1}, m_{2}, m_{12}$, and the $\mathrm{SU}(2)_{L}$ and $\mathrm{U}(1)_{Y}$ gauge couplings $g, g^{\prime}$.

After spontaneous symmetry breaking induced through the neutral components of $H_{1}$ and $H_{2}$ with vacuum expectation values $v_{1}$ and $v_{2}$, respectively, the MSSM Higgs sector contains five physical states: two neutral $C P$-even scalars $\left(h^{0}\right.$ and $\left.H^{0}\right)$, one $C P$-odd pseudoscalar $\left(A^{0}\right)$, and two charged Higgs-boson states $\left(H^{ \pm}\right)$. All quartic coupling constants are related to the electroweak gauge coupling constants, thus imposing various restrictions on the tree-level Higgs-boson masses, couplings, and mixing angles. In particular, all tree-level Higgs-boson parameters can be determined in terms of the mass $M_{A^{0}}$ of the $C P$-odd Higgs boson $\left[M_{A^{0}}^{2}=m_{12}^{2}(\tan \beta+\cot \beta)\right]$, and the ratio of the two Higgsboson vacuum expectation values, $\tan \beta=v_{2} / v_{1}$. The other masses and the mixing angle $\alpha$ for the $C P$-even states $\left(h^{0}, H^{0}\right)$ are then fixed, and the trilinear and quartic selfcouplings of the physical Higgs particles can be predicted.

Our main interest is in the light $h^{0}$ self-couplings, given at the tree level by

$$
\begin{aligned}
\lambda_{h h h}^{0} & =3 \frac{g M_{Z}}{2 c_{W}} \cos 2 \alpha \sin (\beta+\alpha), \\
\lambda_{h h h h}^{0} & =3 \frac{g^{2}}{4 c_{W}^{2}} \cos ^{2} 2 \alpha .
\end{aligned}
$$

In general, they are different from the tree-level couplings of the SM Higgs boson [see Eq. (32) of Sec. III]. However, the situation changes in the decoupling limit of the Higgs sector [3], which implies a particular spectrum with very heavy and quasidegenerate $H^{0}, H^{ \pm}$, and $A^{0}$ Higgs bosons, obeying

$$
\begin{aligned}
& M_{H^{ \pm}}^{2} \simeq M_{A^{0}}^{2}\left[1+\frac{M_{W}^{2}}{M_{A^{0}}^{2}}\right], \\
& M_{H^{0}}^{2} \simeq M_{A^{0}}^{2}\left[1+\left(1-\cos ^{2} 2 \beta\right) \frac{M_{Z}^{2}}{M_{A^{0}}^{2}}\right],
\end{aligned}
$$

and a light $h^{0}$ boson, close to the electroweak scale, with a tree-level mass of

$$
M_{h^{0}}^{\text {tree }} \simeq M_{Z}|\cos 2 \beta| .
$$

This limit also implies $\alpha \rightarrow \beta-\pi / 2$, and thus the tree-level self-couplings (2) tend toward

$$
\begin{aligned}
\lambda_{h h h}^{0} & \simeq 3 \frac{g}{2 M_{W}} M_{h^{0}}^{2 \text { tree }}, \\
\lambda_{h h h h}^{0} & \simeq 3 \frac{g^{2}}{4 M_{W}^{2}} M_{h^{0}}^{2 \text { tree }} .
\end{aligned}
$$

Consequently, the tree-level couplings of the light $C P$-even Higgs boson approach the couplings (32) of a SM Higgs boson with the same mass in the decoupling limit.
However, there are large radiative corrections contributing to the $h^{0}$ self-couplings. The $\mathcal{O}\left(m_{t}^{4}\right)$ top-quark and topsquark contributions were presented recently in [9], with a discussion of decoupling of heavy top-squark particles in the one-loop contributions. Now we will investigate the oneloop contributions to the $h^{0}$ self-couplings originating from the MSSM Higgs sector itself. Thereby, in principle, all kinds of diagrams involving gauge bosons, Goldstone bosons, light and heavy Higgs bosons, have to be taken into account. Some simplifications can be made, however, when one studies the deviations of the $\operatorname{MSSM} h^{0}$ self-couplings from the corresponding SM ones.

(i) The subset of diagrams with only gauge bosons flowing in the loops and the subset of diagrams with both gauge and Goldstone bosons give the same contributions to the $h^{0}$ vertex functions as to the $H_{\mathrm{SM}}$ vertex functions, which we have checked by explicit computation. The only differences come from the extra $\sin (\beta-\alpha)$ factors appearing in the $h^{0}$ case, but these factors tend to 1 in the decoupling limit. Therefore, these kinds of diagrams do not contribute to the differences between $h^{0}$ and $H_{\mathrm{SM}}$ in the decoupling limit, $M_{A^{0}} \gg M_{Z}$, and do not need to be considered in our analysis.

(ii) The contributions from loop diagrams with heavy Higgs bosons $\left(H^{0}, H^{ \pm}, A^{0}\right)$ together with gauge bosons always go with factors $\cos (\beta-\alpha)$ and, therefore, they vanish in the large $M_{A^{0}}$ limit. We have also checked this explicitly. We thus do not need to consider these diagrams here either.

(iii) Diagrams involving loops with MSSM heavy Higgs bosons together with Goldstone bosons or the lightest Higgs boson do not appear in the SM. Contrary to the previous case, the vertices in these Feynman diagrams are not proportional to $\cos (\beta-\alpha)$ and they do not vanish in the decoupling limit. These diagrams must therefore be included explicitly in our computation. Moreover, the purely MSSM heavy Higgs-boson one-loop contributions are obviously an exclusive property of the MSSM and thus they have to be taken into account as well. In addition, there are contributions from diagrams involving just Goldstone bosons and the lightest Higgs boson in the loops. A priori, they do not look the same in both models. However, as we will see in course of the discussion, they converge to the SM ones in the $M_{A^{0}} \gg M_{Z}$ limit (see Secs. II A and III).

For a transparent discussion, we present the details of the computation in the following subsections. First, we will give the one-loop results for the unrenormalized vertex functions of the lightest Higgs boson $h^{0}$, by considering the limit $M_{A^{0}} \gg M_{Z}$ in the MSSM Higgs sector. Then, we give a discussion of the on-shell renormalization scheme for the MSSM Higgs bosons and list the expressions for the $h^{0}$ vertex function counterterms and the explicit results for the renormalization constants in the large $M_{A^{0}}$ limit (Sec. II B). Finally, the renormalized vertex results are given at the end of Sec. II B.

\section{A. Higgs boson self-couplings in the large- $M_{A^{0}}$ limit}

From now on, the general results for the $n$-point ( $n$ $=1, \ldots, 4)$ renormalized vertex functions will be summarized by the generic expression 
<smiles>O=P1(I)CCSCC1</smiles>
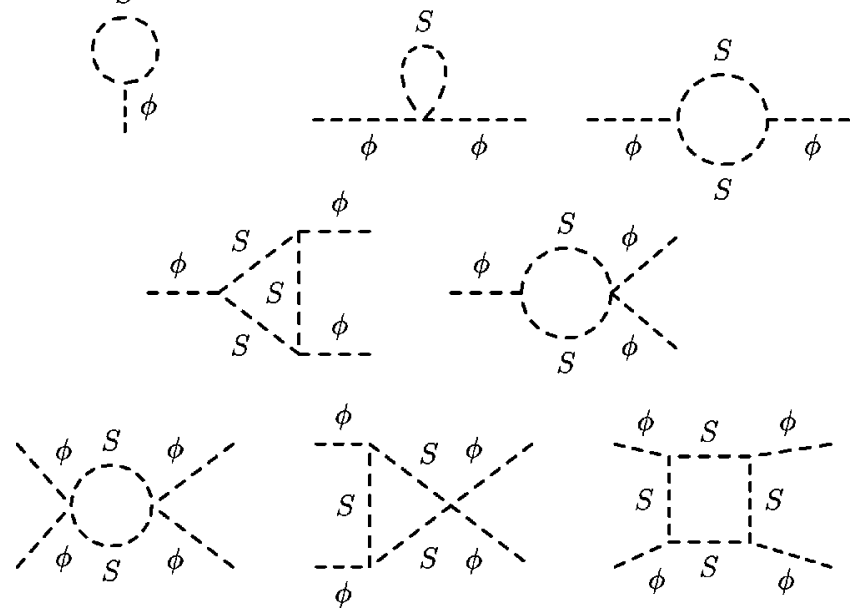

FIG. 1. Generic one-loop diagrams contributing to the $n$-point $(n=1,2,3,4)$ functions of the Higgs boson. Here $\phi \equiv h^{0}(\phi$ $\left.\equiv H_{S M}\right)$ in the MSSM (SM) case and correspondingly $S$ $\equiv h^{0}, G^{0}, G^{ \pm}, H^{0}, H^{ \pm}, A^{0}\left(S \equiv H_{S M}, G^{0}, G^{ \pm}\right)$.

$$
\Gamma_{R H}^{(n)}=\Gamma_{0 H}^{(n)}+\Delta \Gamma_{R H}^{(n)}=\Gamma_{0 H}^{(n)}+\Delta \Gamma_{H}^{(n)}+\delta \Gamma_{H}^{(n)},
$$

where the subscript $R$ denotes renormalized functions, the subscript 0 refers to the tree-level functions, the one-loop contributions are summarized in $\Delta \Gamma_{H}^{(n)}$, and $\delta \Gamma_{H}^{(n)}$ represent the counterterm contributions. The sum of these two last contributions is denoted by $\Delta \Gamma_{R H}^{(n)}$. Here $H$ refers to the external Higgs-boson particle, which corresponds to the lightest $C P$-even Higgs boson $H \equiv h^{0}$ in the MSSM and to the SM Higgs boson $H \equiv H_{\mathrm{SM}}$ in the SM case. The tree-level functions in the MSSM, $\Gamma_{0 h^{0}}^{(n)}$, for $n=3$ and $n=4$, are the trilinear and quartic $h^{0}$ Higgs self-couplings, already given in Eq. (2), and $\Gamma_{0 h^{0}}^{(2)}=-\left(q^{2}-M_{h^{0}}^{2 \text { tree }}\right)$. Obviously, $\Gamma_{0 H}^{(1)}=0$.

We will present in this subsection the results for the oneloop contributions $\Delta \Gamma_{h^{0}}^{(n)}$ that come from the diagrams shown generically in Fig. 1. The computation has been performed by the diagrammatic method utilizing FEYNARTS 3 and FORMCALC [15], and the results are expressed in terms of the standard one-loop integrals [16]. We have made the computation in a general $R_{\xi}$ gauge and we have used dimensional regularization to compute the one-loop integrals. Some details of how to compute the integrals in the large mass limit $M_{A^{0}} \gg M_{Z}$ can be seen in [11]. In the decoupling limit, the heavy Higgs-boson masses have similar size, up to terms of
$\mathcal{O}\left(M_{Z}^{2} / M_{A^{0}}^{2}\right)$ [see Eq. (3)], and correspondingly the $\alpha$ angle expansion leads to $\mathcal{O}\left(M_{Z}^{2} / M_{A^{0}}^{2}\right)$ terms, such that

$$
\begin{aligned}
& \sin (\beta-\alpha) \simeq 1, \quad \cos (\beta-\alpha) \simeq \frac{M_{Z}^{2}}{M_{A^{0}}^{2}} S_{2 \beta} C_{2 \beta}, \\
& \sin (\beta+\alpha) \simeq-C_{2 \beta}\left(1-\frac{M_{Z}^{2}}{M_{A^{0}}^{2}} S_{2 \beta}^{2}\right), \\
& \cos (\beta+\alpha) \simeq S_{2 \beta}\left(1+\frac{M_{Z}^{2}}{M_{A^{0}}^{2}} C_{2 \beta}^{2}\right), \\
& \sin 2 \alpha \simeq-S_{2 \beta}\left(1+2 \frac{M_{Z}^{2}}{M_{A^{0}}^{2}} C_{2 \beta}^{2}\right), \\
& \cos 2 \alpha \simeq-C_{2 \beta}\left(1-2 \frac{M_{Z}^{2}}{M_{A^{0}}^{2}} S_{2 \beta}^{2}\right) \text {. }
\end{aligned}
$$

Here, and throughout this paper, $C_{2 \beta} \equiv \cos 2 \beta$ and $S_{2 \beta}$ $\equiv \sin 2 \beta$.

Finally, with the explicit results for the one-loop integrals, we obtain the contributions to the $n$-point functions. For a later comparison with the SM, it is convenient to split the results according to

$$
\Delta \Gamma_{h^{0}}^{(n)}=\Delta \Gamma_{h^{0}}^{(n)^{\text {light }}}+\Delta \Gamma_{h^{0}}^{(n)^{\text {mixed }}}+\Delta \Gamma_{h^{0}}^{(n)^{\text {heavy }}}
$$

where $\Delta \Gamma_{h^{0}}^{(n)^{\text {light }}}$ refers to one-loop diagrams with Goldstone bosons $\left(G^{0}, G^{ \pm}\right)$and the lightest Higgs boson $h^{0}, \Delta \Gamma_{h^{0}}^{(n)^{\text {mixed }}}$ refers to the one-loop diagrams involving heavy Higgs particles $\left(H^{0}, H^{ \pm}, A^{0}\right)$ together with the $h^{0}$ boson or Goldstone bosons flowing in the loops, and, finally, $\Delta \Gamma_{h^{0}}^{(n)^{\text {heavy }}}$ refers to the diagrams with MSSM purely heavy Higgs contributions (only $H^{0}, H^{ \pm}$, and $A^{0}$ in the one-loop diagrams).

We first list the light one-loop vertex terms. Note that for our study both the momenta of the external $h^{0}$ lines and the masses of $h^{0}, Z, W^{ \pm}$are quantities to be considered at the low-energy scales below $M_{A^{0}}$. The corresponding subset of diagrams is depicted generically in Fig. 1 for the MSSM case by setting $\phi \equiv h^{0}$ and $S \equiv h^{0}, G^{0}, G^{ \pm}$. The contributions from the first three-point diagram and from the last two four-point diagrams are UV finite. The residual diagrams give both a finite contribution and a divergent part. Expressed in terms of the scalar one-loop integrals [16] in the convention of [17], we have, for $\Delta \Gamma_{h^{0}}^{(n)^{\text {light }}}$ in the large $M_{A}$ limit,

$$
\begin{aligned}
\Delta \Gamma_{h^{0}}^{(1) \text { light }}= & \frac{g M_{Z}}{64 \pi^{2} c_{W}} C_{2 \beta}^{2}\left\{3 A_{0}\left(M_{h^{0}}^{2}\right)+A_{0}\left(\xi M_{Z}^{2}\right)+2 A_{0}\left(\xi M_{W}^{2}\right)\right\}, \\
\Delta \Gamma_{h^{0}}^{(2) \text { light }}= & \frac{g^{2}}{128 \pi^{2} c_{W}^{2}} C_{2 \beta}^{2}\left\{3 A_{0}\left(M_{h^{0}}^{2}\right)+A_{0}\left(\xi M_{Z}^{2}\right)+2 A_{0}\left(\xi M_{W}^{2}\right)+M_{Z}^{2} C_{2 \beta}^{2}\left[9 B_{0}\left(q^{2}, M_{h^{0}}^{2}, M_{h^{0}}^{2}\right)+B_{0}\left(q^{2}, \xi M_{Z}^{2}, \xi M_{Z}^{2}\right)\right.\right. \\
& \left.\left.+2 B_{0}\left(q^{2}, \xi M_{W}^{2}, \xi M_{W}^{2}\right)\right]\right\},
\end{aligned}
$$




$$
\begin{aligned}
\Delta \Gamma_{h^{0}}^{(3) \text { light }}= & \frac{g^{3}}{256 \pi^{2} c_{W}^{3}} M_{Z} C_{2 \beta}^{4}\left\{\left[9 B_{0}\left(q^{2}, M_{h^{0}}^{2}, M_{h^{0}}^{2}\right)+B_{0}\left(q^{2}, \xi M_{Z}^{2}, \xi M_{Z}^{2}\right)+2 B_{0}\left(q^{2}, \xi M_{W}^{2}, \xi M_{W}^{2}\right)+(q \rightarrow p)+(q \rightarrow r)\right]\right. \\
& +2 M_{Z}^{2} C_{2 \beta}^{2}\left[27 C_{0}\left(q^{2}, p^{2}, r^{2}, M_{h^{0}}^{2}, M_{h^{0}}^{2}, M_{h^{0}}^{2}\right)+C_{0}\left(q^{2}, p^{2}, r^{2}, \xi M_{Z}^{2}, \xi M_{Z}^{2}, \xi M_{Z}^{2}\right)\right. \\
& \left.\left.+2 C_{0}\left(q^{2}, p^{2}, r^{2}, \xi M_{W}^{2}, \xi M_{W}^{2}, \xi M_{W}^{2}\right)\right]\right\}, \\
\Delta \Gamma_{h^{0}}^{(4)}= & \frac{g^{4}}{512 \pi^{2} c_{W}^{4}} C_{2 \beta}^{4}\left\{\left[9 B_{0}\left((q+p)^{2}, M_{h^{0}}^{2}, M_{h^{0}}^{2}\right)+B_{0}\left((q+p)^{2}, \xi M_{Z}^{2}, \xi M_{Z}^{2}\right)+B_{0}\left((q+p)^{2}, \xi M_{W}^{2}, \xi M_{W}^{2}\right)+(p \rightarrow r)\right.\right. \\
& +(p \rightarrow t)]+2 M_{Z}^{2} C_{2 \beta}^{2}\left[27 C_{0}\left(q^{2}, p^{2},(q+p)^{2}, M_{h^{0}}^{2}, M_{h^{0}}^{2}, M_{h^{0}}^{2}\right)+C_{0}\left(q^{2}, p^{2},(q+p)^{2}, \xi M_{Z}^{2}, \xi M_{Z}^{2}, \xi M_{Z}^{2}\right)\right. \\
& \left.+2 C_{0}\left(q^{2}, p^{2},(q+p)^{2}, \xi M_{W}^{2}, \xi M_{W}^{2}, \xi M_{W}^{2}\right)(p \rightarrow r)+(p \rightarrow t)+(q \rightarrow t, p \rightarrow r)+(q \rightarrow p, p \rightarrow r)+(q \rightarrow p, p \rightarrow t)\right] \\
& +2 M_{Z}^{4} C_{2 \beta}^{4}\left[81 D_{0}\left(q^{2}, p^{2}, r^{2}, t^{2},(q+p)^{2},(p+r)^{2}, M_{h^{0}}^{2}, M_{h^{0}}^{2}, M_{h^{0}}^{2}, M_{h^{0}}^{2}\right)\right. \\
& +D_{0}\left(q^{2}, p^{2}, r^{2}, t^{2},(q+p)^{2},(p+r)^{2}, \xi M_{Z}^{2}, \xi M_{Z}^{2}, \xi M_{Z}^{2}, \xi M_{Z}^{2}\right) \\
& \left.\left.+2 D_{0}\left(q^{2}, p^{2}, r^{2}, t^{2},(q+p)^{2},(p+r)^{2}, \xi M_{W}^{2}, \xi M_{W}^{2}, \xi M_{W}^{2}, \xi M_{W}^{2}\right)+(r \leftrightarrow t)+(p \leftrightarrow r)\right]\right\} .
\end{aligned}
$$

Here $q, p, r$, and $t$ denote the external momenta, $\xi$ is the gauge parameter, and $c_{W}=\cos \theta_{W}$. Notice that these contributions are $\xi$-gauge dependent. In addition, since they show an explicit dependence on $\beta$, they are different from the SM ones for arbitrary $\tan \beta$ values. However, as we will see explicitly in Sec. III [see Eq. (33)], they converge to the SM ones in the $M_{A^{0}} \gg M_{Z}$ limit. Thus, by identifying the light $C P$-even Higgs-boson mass $M_{h^{0}}^{\text {tree }} \simeq M_{Z}\left|C_{2 \beta}\right|$ with the SM Higgs-boson mass $M_{H_{\mathrm{SM}}}^{\text {tree }}$, the contributions (9) acquire the structure of the unrenormalized SM one-loop vertex functions (33). Therefore, we conclude that the contributions involving only Goldstone bosons and the lightest Higgs boson in the loops are the same in both models in the $M_{A}{ }^{0} \gg M_{Z}$ limit. This is equivalent to stating that the difference between the one-loop unrenormalized $n$-point functions of the two theories in the decoupling limit originates only from diagrams including at least one heavy MSSM Higgs particle. These contributions correspond to $\Delta \Gamma_{h^{0}}^{(n)}$ heavy and $\Delta \Gamma_{h^{0}}^{(n) \text { mixed }}$, which read as follows:

$$
\begin{aligned}
\Delta \Gamma_{h^{0}}^{(1)^{\text {heavy }}=}= & \frac{g M_{Z}}{32 \pi^{2} c_{W}}\left\{M_{A^{0}}^{2}\left(1+2 c_{W}^{2}-3 C_{2 \beta}^{2}\right)\left(\Delta_{\epsilon}+1-\log \frac{M_{A^{0}}^{2}}{\mu_{0}^{2}}\right)\right. \\
& \left.+M_{Z}^{2}\left[6 C_{2 \beta^{2}}^{2} S_{2 \beta}^{2}+\frac{1}{2}\left(2-9 C_{2 \beta}^{4}+4 c_{W}^{4}+7 C_{2 \beta}^{2}-2 C_{2 \beta}^{2} c_{W}^{2}\right)\left(\Delta_{\epsilon}-\log \frac{M_{A^{0}}^{2}}{\mu_{0}^{2}}\right)\right]\right\}, \\
\Delta \Gamma_{h^{0}}^{(2)^{\text {heavy }}}= & \frac{g^{2}}{64 \pi^{2} c_{W}^{2}}\left\{M_{A^{0}}^{2}\left(1+2 c_{W}^{2}-3 C_{2 \beta}^{2}\right)\left(\Delta_{\epsilon}+1-\log \frac{M_{A^{0}}^{2}}{\mu_{0}^{2}}\right)\right. \\
& \left.+M_{Z}^{2}\left[9 C_{2 \beta^{3}}^{2} S_{2 \beta}^{2}+\frac{1}{2}\left(6-3 C_{2 \beta}^{4}+12 c_{W}^{4}+C_{2 \beta}^{2}-10 C_{2 \beta}^{2} c_{W}^{2}\right)\left(\Delta_{\epsilon}-\log \frac{M_{A^{0}}^{2}}{\mu_{0}^{2}}\right)\right]\right\}, \\
\Delta \Gamma_{h^{0}}^{(2) \text { mixed }}= & \frac{g^{2}}{32 \pi^{2} c_{W}^{2}} M_{Z}^{2} 6 C_{2 \beta}^{2} S_{2 \beta}^{2}\left(\Delta_{\epsilon}+1-\log \frac{M_{A^{0}}^{2}}{\mu_{0}^{2}}\right), \\
\Delta \Gamma_{h^{0}}^{(3) \text { heavy }}= & \frac{3 g^{3}}{64 \pi^{2} c_{W}^{3}} M_{Z}\left(1+3 C_{2 \beta}^{4}+2 c_{W}^{4}-3 C_{2 \beta}^{2}-2 C_{2 \beta}^{2} c_{W}^{2}\right)\left(\Delta_{\epsilon}-\log \frac{M_{A^{0}}^{2}}{\mu_{0}^{2}}\right), \\
\Delta \Gamma_{h^{0}}^{(3)^{\text {mixed }}=} & \frac{3 g^{3}}{64 \pi^{2} c_{W}^{3}} M_{Z} 6 C_{2 \beta}^{2} S_{2 \beta}^{2}\left(\Delta_{\epsilon}+1-\log \frac{M_{A^{0}}^{2}}{\mu_{0}^{2}}\right),
\end{aligned}
$$




$$
\begin{aligned}
& \Delta \Gamma_{h^{0}}^{(4)^{\text {heavy }}}=\frac{3 g^{4}}{128 \pi^{2} c_{W}^{4}}\left(1+3 C_{2 \beta}^{4}+2 c_{W}^{4}-3 C_{2 \beta}^{2}-2 C_{2 \beta}^{2} c_{W}^{2}\right)\left(\Delta_{\epsilon}-\log \frac{M_{A^{0}}^{2}}{\mu_{0}^{2}}\right), \\
& \Delta \Gamma_{h^{0}}^{(4)^{\text {mixed }}}=\frac{3 g^{4}}{128 \pi^{2} c_{W}^{4}} 6 C_{2 \beta}^{2} S_{2 \beta}^{2}\left(\Delta_{\epsilon}+1-\log \frac{M_{A^{0}}^{2}}{\mu_{0}^{2}}\right) .
\end{aligned}
$$

Obviously, $\Delta \Gamma_{h^{0}}^{(1)^{\text {mixed }}}=0$. Here $\mu_{0}$ denotes the scale of dimensional regularization and the singular $\Delta_{\epsilon}$ term is defined, as usual, by

$$
\Delta_{\epsilon}=\frac{2}{\epsilon}-\gamma_{\epsilon}+\log (4 \pi), \quad \epsilon=4-D .
$$

Terms that are suppressed by inverse powers of the heavy mass $M_{A^{0}}$ and thus vanish in the decoupling limit are dropped in the expressions given above. Contrary to the oneloop contributions from diagrams with Goldstone bosons and the lightest $h^{0}$ Higgs particle (9), the above $h^{0}$ vertex function contributions are $\xi$-gauge independent. The Feynman diagrams contributing to the one-loop MSSM heavy Higgsboson sector part, $\Delta \Gamma_{h^{0}}^{(n)^{\text {heavy }}}(n=1, \ldots, 4)$, appearing in Eqs. (10)-(13) can be extracted from Fig. 1 by choosing $\phi$ $\equiv h^{0}$ and $S \equiv H^{0}, H^{ \pm}, A^{0}$. The contributions from the first diagram in the three-point function and from the last two diagrams in the four-point function are finite and vanish in the $M_{A^{0}} \gg M_{Z}$ limit. The remaining diagrams are UV divergent and contain a logarithmic dependence on the heavy pseudoscalar mass $M_{A^{0}}$ and, for $n=1,2$, a quadratic dependence on $M_{A^{0}}$ as well. In contrast, the mixed diagrams do not give $M_{A^{0}}^{2}$ terms, but they are logarithmically dependent on $M_{A^{0}}$. The corresponding specific Feynman diagrams are obtained from Fig. 1 by taking $\phi \equiv h^{0}$ and accordingly to the light and heavy particles that can be flowing in the loops, $S$ $\equiv h^{0}, G^{0}, G^{ \pm}, H^{0}, H^{ \pm}, A^{0}$. More specifically, the mixed diagrams that give contributions different from zero in the $d e$ coupling limit correspond to the third, fifth, and sixth diagrams in Fig. 1, with $h^{0}$ and $H^{0}\left(S \equiv h^{0}, H^{0}\right), G^{0}$, and $A^{0}$ $\left(S \equiv G^{0}, A^{0}\right)$, and $G^{ \pm}$and $H^{ \pm}\left(S \equiv G^{ \pm}, H^{ \pm}\right)$, in the two internal propagators of the loops.

Let us remark that, in these results for the unrenormalized vertex functions, all the potential nondecoupling effects of the heavy Higgs MSSM particles manifest as some divergent contributions in $D=4$ and some finite contributions, one of which is logarithmically dependent on the heavy pseudoscalar Higgs-boson mass $M_{A^{0}}$ and the other one is quadratically dependent on $M_{A^{0}}$. Obviously, all the results displayed up to now are, in general, UV divergent. In order to get finite 1PI Green functions and finite predictions for physical observables, renormalization has to be performed by adding appropriate counterterms. This is the subject of the next subsection.

\section{B. Renormalization in the MSSM}

For a systematic one-loop computation, the free parameters of the Higgs potential $m_{1}^{2}, m_{2}^{2}, m_{12}^{2}, g, g^{\prime}$ and the two vacua $v_{1}, v_{2}$ are replaced by the corresponding renormalized parameters plus counterterms. This transforms the potential $V$ into $V+\delta V$, where $V$ is expressed in terms of the renormalized parameters, and $\delta V$ is the counterterm potential. By using the standard renormalization procedure $[18,19]$

$$
\begin{aligned}
m_{i}^{2} & \rightarrow Z_{H_{i}}^{-1}\left(m_{i}^{2}+\delta m_{i}^{2}\right), \\
m_{12}^{2} & \rightarrow Z_{H_{1}}^{-1 / 2} Z_{H_{2}}^{-1 / 2}\left(m_{12}^{2}+\delta m_{12}^{2}\right), \\
v_{i} & \rightarrow Z_{H_{i}}^{1 / 2}\left(v_{i}-\delta v_{i}\right), \\
g & \rightarrow Z_{1}^{W} Z_{2}^{W-3 / 2} g, \quad g^{\prime} \rightarrow Z_{1}^{B} Z_{2}^{B-3 / 2} g^{\prime},
\end{aligned}
$$

with Higgs-field renormalization constants $\delta Z_{H_{i}}$, and by using the minimum condition on the potential at tree level, we obtain the counterterms for the $n$-point $(n=1, \ldots, 4)$ vertex functions. The results in the decoupling limit are

$$
\begin{aligned}
\delta \Gamma_{h^{0}}^{(1)}= & \frac{g M_{Z}}{2 c_{W}} C_{2 \beta} v^{2}\left(\sin ^{2} \beta \delta Z_{H_{2}}-\cos ^{2} \beta \delta Z_{H_{1}}\right)-v \delta M_{12}^{2}+\frac{1}{4} \frac{g^{2}}{c_{W}^{2}} v^{2} C_{2 \beta}^{2} \delta v-\frac{1}{8} v^{3} C_{2 \beta}^{2} \delta G^{2} \\
& +C_{2 \beta} S_{2 \beta} \frac{M_{Z}^{2}}{M_{A^{0}}^{2}}\left[\frac{g^{2}}{16 c_{W}^{2}} S_{2 \beta} v^{3}\left[\delta Z_{H_{2}}\left(2 C_{2 \beta}-1\right)+\delta Z_{H_{1}}\left(2 C_{2 \beta}+1\right)\right]\right. \\
& \left.-v \delta C_{12}^{2}-\frac{g^{2}}{4 c_{W}^{2}} C_{2 \beta} S_{2 \beta} v^{2} \delta v+\frac{1}{8} v^{3} C_{2 \beta} S_{2 \beta} \delta G^{2}\right],
\end{aligned}
$$




$$
\begin{aligned}
\delta \Gamma_{h^{0}}^{(2)}= & q^{2}\left[\left(\sin ^{2} \beta \delta Z_{H_{2}}+\cos ^{2} \beta \delta Z_{H_{1}}\right)+\frac{M_{Z}^{2}}{M_{A^{0}}^{2}} C_{2 \beta} S_{2 \beta}^{2}\left(\delta Z_{H_{2}}-\delta Z_{H_{1}}\right)\right]+\frac{3}{4}\left[C_{2 \beta} v^{2} \frac{g^{2}}{c_{W}^{2}}\left(\sin ^{2} \beta \delta Z_{H_{2}}-\cos ^{2} \beta \delta Z_{H_{1}}\right)\right. \\
& \left.-\frac{4}{3} \delta M_{12}^{2}+\frac{g^{2}}{c_{W}^{2}} C_{2 \beta}^{2} v \delta v-\frac{v^{2}}{2} C_{2 \beta}^{2} \delta G^{2}\right]+C_{2 \beta} S_{2 \beta} \frac{M_{Z}^{2}}{M_{A^{0}}^{2}}\left[\frac{3 g^{2}}{8 c_{W}^{2}} S_{2 \beta} v^{2}\left[\delta Z_{H_{2}}\left(2 C_{2 \beta}-1\right)+\delta Z_{H_{1}}\left(2 C_{2 \beta}+1\right)\right]\right. \\
& \left.-2 \delta C_{12}^{2}-\frac{3 g^{2}}{2 c_{W}^{2}} C_{2 \beta} S_{2 \beta} v \delta v+\frac{3}{4} v^{2} C_{2 \beta} S_{2 \beta} \delta G^{2}\right], \\
\delta \Gamma_{h^{0}}^{(3)}= & \frac{3}{4} C_{2 \beta}\left[2 v \frac{g^{2}}{c_{W}^{2}}\left(\sin ^{2} \beta \delta Z_{H_{2}}-\cos ^{2} \beta \delta Z_{H_{1}}\right)+\frac{g^{2}}{c_{W}^{2}} C_{2 \beta} \delta v-v C_{2 \beta} \delta G^{2}\right] \\
& +\frac{9}{4} C_{2 \beta} S_{2 \beta} \frac{M_{Z}^{2}}{M_{A^{0}}{ }^{2}}\left[\frac{g^{2}}{4 c_{W}^{2}} S_{2 \beta} v\left[\delta Z_{H_{2}}\left(2 C_{2 \beta}-1\right)+\delta Z_{H_{1}}\left(2 C_{2 \beta}+1\right)\right]-\frac{g^{2}}{c_{W}^{2}} C_{2 \beta} S_{2 \beta} \delta v+v C_{2 \beta} S_{2 \beta} \delta G^{2}\right], \\
\delta \Gamma_{h^{0}}^{(4)}= & \frac{3}{4} C_{2 \beta}\left[2 \frac{g^{2}}{c_{W}^{2}}\left(\sin ^{2} \beta \delta Z_{H_{2}}-\cos ^{2} \beta \delta Z_{H_{1}}\right)-C_{2 \beta} \delta G^{2}\right]+\frac{9}{4} C_{2 \beta} S_{2 \beta} \frac{M_{Z}^{2}}{M_{A^{0}}^{2}}\left[\frac { g ^ { 2 } } { 4 c _ { W } ^ { 2 } } S _ { 2 \beta } \left[\delta Z_{H_{2}}\left(2 C_{2 \beta}-1\right)\right.\right. \\
& \left.\left.+\delta Z_{H_{1}}\left(2 C_{2 \beta}+1\right)\right]+C_{2 \beta} S_{2 \beta} \delta G^{2}\right] .
\end{aligned}
$$

Here all $\mathcal{O}\left(M_{Z}^{2} / M_{A^{0}}^{2}\right)$ contributions are still explicitly included. We have introduced the abbreviations

$$
\begin{aligned}
\delta G^{2} & \equiv \delta g^{2}+\delta g^{\prime 2}=g^{2}\left(2 \delta Z_{1}^{W}-3 \delta Z_{2}^{W}\right)-g^{\prime 2} \delta Z_{2}^{B}, \\
\delta M_{12}^{2} & \equiv \cos ^{2} \beta \delta m_{1}^{2}+\sin ^{2} \beta \delta m_{2}^{2}+S_{2 \beta} \delta m_{12}^{2}, \\
v \delta v & =v_{1} \delta v_{1}+v_{2} \delta v_{2} \quad \text { with } \quad v^{2}=v_{1}^{2}+v_{2}^{2}, \\
\delta C_{12}^{2} & \equiv C_{2 \beta} \delta m_{12}^{2}+\frac{S_{2 \beta}}{2}\left(\delta m_{2}^{2}-\delta m_{1}^{2}\right) .
\end{aligned}
$$

Correspondingly, the tadpole counterterm for the $H^{0}$ Higgs boson, $\delta \Gamma_{H^{0}}^{(1)}$, and the counterterm for the pseudoscalar two-point function, $\delta \Gamma_{A^{0}}^{(2)}$, which are necessary for the MSSM on-shell renormalization, are given, in the decoupling limit, by

$$
\begin{aligned}
\delta \Gamma_{H^{0}}^{(1)}= & -\frac{g M_{Z}}{8 c_{W}} S_{2 \beta} v^{2}\left[\left(-1+C_{2 \beta}\right) \delta Z_{H_{2}}+\left(1+C_{2 \beta}\right) \delta Z_{H_{1}}\right]+v \delta C_{12}^{2}+\frac{1}{4} \frac{g^{2}}{c_{W}^{2}} v^{2} C_{2 \beta} S_{2 \beta} \delta v-\frac{1}{8} v^{3} C_{2 \beta} S_{2 \beta} \delta G^{2} \\
& -C_{2 \beta} S_{2 \beta} \frac{M_{Z}^{2}}{M_{A^{0}}^{2}}\left[\frac{g^{2}}{4 c_{W}^{2}}\left(1-2 C_{2 \beta}^{2}\right) v^{3}\left(\sin ^{2} \beta \delta Z_{H_{2}}-\cos ^{2} \beta \delta Z_{H_{1}}\right)+v \delta M_{12}^{2}-\frac{g^{2}}{4 c_{W}^{2}} C_{2 \beta}^{2} v^{2} \delta v+\frac{1}{8} v^{3} C_{2 \beta}^{2} \delta G^{2}\right], \\
\delta \Gamma_{A^{0}}^{(2)}= & q^{2}\left(\sin ^{2} \beta \delta Z_{H_{1}}+\cos ^{2} \beta \delta Z_{H_{2}}\right)-\frac{1}{2}\left(\sin ^{2} \beta \delta m_{1}^{2}+\cos ^{2} \beta \delta m_{2}^{2}-\sin 2 \beta \delta m_{12}^{2}\right)+\frac{1}{8} \frac{g^{2}}{c_{W}^{2}} v^{2} C_{2 \beta}^{2} \\
& \times\left(\frac{c_{W}^{2}}{g^{2}} \delta G^{2}+\delta Z_{H_{1}}+\delta Z_{H_{2}}-2 \frac{\delta v}{v}\right) .
\end{aligned}
$$

We note that no $\mathcal{O}\left(M_{A^{0}}^{2}\right)$ contributions to the renormalization constants $\delta G^{2}, \delta v$, and $\delta Z_{H_{i}}(i=1,2)$ exist. Therefore, terms of the type $\mathcal{O}\left(M_{Z}^{2} / M_{A^{0}}^{2}\right) \cdot \delta G^{2}$, $\mathcal{O}\left(M_{Z}^{2} / M_{A^{0}}^{2}\right) \cdot \delta v$, or $\mathcal{O}\left(M_{Z}^{2} / M_{A^{0}}^{2}\right) \cdot \delta Z_{H_{i}}$ in Eqs. (16) and (18) can be safely neglected.
In the on-shell scheme, the counterterms are fixed by imposing the following renormalization conditions $[18,21]$ : the on-shell conditions for $M_{W, Z}$ and the electric charge $e$, as in the SM; the on-shell condition for the $A^{0}$ boson with the pole mass $M_{A}$; the tadpole conditions for vanishing renormalized tadpoles for both the $H^{0}$ and $h^{0}$ Higgs fields, i.e., the sum of 
the one-loop tadpole diagrams for $H^{0}, h^{0}$ and the corresponding tadpole counterterms is equal to zero; and the renormalization of $\tan \beta$ in such a way that the relation $\tan \beta$ $=v_{2} / v_{1}$ is valid for the true one-loop Higgs minima.

Notice that the above condition for vanishing renormalized tadpole diagrams ensures that $v_{1}, v_{2}$ determine the minimum of the one-loop potential. The relation $\tan \beta=v_{2} / v_{1}$ in terms of the "true vacua" is maintained by the condition $\delta v_{1} / v_{1}=\delta v_{2} / v_{2}$. By the above set of conditions, the input for the MSSM Higgs sector is fixed by the $A^{0}$ pole mass $M_{A}$ and $\tan \beta$, together with the standard gauge-sector input $M_{W, Z}$ and $e$.

In order to compute the renormalization constants $\delta Z_{H_{1}}$, $\delta Z_{\mathrm{H}_{2}}, \delta G^{2}$, and $\delta v$, we express them in terms of the vector boson self-energies, the $A^{0}$-boson self-energy, and the $A^{0} Z$ nondiagonal self-energy [18]:

$$
\begin{aligned}
\delta Z_{H_{1}}= & -\Sigma_{A^{0}}^{\prime}\left(M_{A^{0}}^{2}\right)-\frac{\cot \beta}{M_{Z}} \Sigma_{A^{0} Z}\left(M_{A^{0}}^{2}\right), \\
\delta Z_{H_{2}}= & -\Sigma_{A^{0}}^{\prime}\left(M_{A^{0}}^{2}\right)+\frac{\tan \beta}{M_{Z}} \Sigma_{A^{0} Z}\left(M_{A^{0}}^{2}\right), \\
\delta G^{2}= & \frac{g^{2}}{c_{W}^{2}}\left[\Sigma_{\gamma}^{\prime}(0)-2 \frac{s_{W}}{c_{W}} \frac{\Sigma_{\gamma Z}(0)}{M_{Z}^{2}}\right. \\
& \left.-\frac{c_{W}^{2}-s_{W}^{2}}{s_{W}^{2}}\left(\frac{\Sigma_{Z}\left(M_{Z}^{2}\right)}{M_{Z}^{2}}-\frac{\Sigma_{W}\left(M_{W}^{2}\right)}{M_{W}^{2}}\right)\right],
\end{aligned}
$$

$$
\begin{aligned}
2 \frac{\delta v}{v}= & -\Sigma_{A^{0}}^{\prime}\left(M_{A^{0}}^{2}\right)+\frac{\tan \beta-\cot \beta}{M_{Z}} \Sigma_{A^{0} Z}\left(M_{A^{0}}^{2}\right) \\
& +\Sigma_{\gamma}^{\prime}(0)-2 \frac{s_{W}}{c_{W}} \frac{\Sigma_{\gamma Z}(0)}{M_{Z}^{2}}-\frac{c_{W}^{2}}{s_{W}^{2}} \frac{\Sigma_{Z}\left(M_{Z}^{2}\right)}{M_{Z}^{2}} \\
& +\frac{c_{W}^{2}-s_{W}^{2}}{s_{W}^{2}} \frac{\Sigma_{W}\left(M_{W}^{2}\right)}{M_{W}^{2}} .
\end{aligned}
$$

Partial results for the one-loop contributions to the vector boson self-energies can be extracted from the last article in Ref. [11] or from the first article in Ref. [18]. We have recalculated explicitly all the self-energies that appear in Eq. (20), and we have checked that our results agree with previous ones in the literature. Here we do not present the intermediate results, but list only the final expressions for the counterterms.

First, we found that $\delta Z_{H_{1,2}}$ get contributions that are suppressed by inverse powers of the heavy mass $M_{A^{0}}$. However, such terms of order $\mathcal{O}\left(M_{Z}^{2} / M_{A^{0}}^{2}\right)$ to $\delta Z_{H_{1,2}}$ are relevant in order to implement consistently the $A^{0}$-boson on-shell condition $\Delta \Gamma_{A^{0}}^{(2)}\left(M_{A^{0}}^{2}\right)+\delta \Gamma_{A^{0}}^{(2)}\left(M_{A^{0}}^{2}\right)=0$. Their expressions are given explicitly in the Appendix.

The various contributions to $\delta v$ and $\delta G^{2}$ are split again into "light" and "heavy." The light ones originate from diagrams involving Goldstone bosons and the lightest $h^{0}$ Higgs boson in the loops,

$$
\begin{aligned}
& \frac{\delta v_{\text {light }}}{v}=-\frac{g^{2}}{128 c_{W}^{2} s_{W}^{2} \pi^{2}} \frac{1}{M_{Z}^{2}}\left(s_{W}^{2}\left[A_{0}\left(M_{h^{0}}^{2}\right)+A_{0}\left(\xi M_{Z}^{2}\right)\right]-2\left(1-c_{W}^{2}-4 c_{W}^{4}+4 c_{W}^{6}\right) A_{0}\left(\xi M_{W}^{2}\right)+\frac{4}{3} M_{Z}^{2} c_{W}^{2} s_{W}^{4} B_{0}\left(0, \xi M_{W}^{2}, \xi M_{W}^{2}\right)\right. \\
& +4 c_{W}^{2}\left\{\left(1-2 c_{W}^{2}\right)^{2} B_{22}\left(M_{Z}^{2}, \xi M_{W}^{2}, \xi M_{W}^{2}\right)+B_{22}\left(M_{Z}^{2}, M_{h^{0}}^{2}, \xi M_{Z}^{2}\right)+\left(1-2 c_{W}^{2}\right)\left[B_{22}\left(M_{W}^{2}, M_{h^{0}}^{2}, \xi M_{W}^{2}\right)\right.\right. \\
& \left.\left.\left.+B_{22}\left(M_{W}^{2}, \xi M_{Z}^{2}, \xi M_{W}^{2}\right)\right]\right\}\right), \\
& \delta G^{2 \text { light }}=\frac{g^{4}}{16 c_{W}^{4} s_{W}^{2} \pi^{2}} \frac{1}{M_{Z}^{2}}\left\{2 c_{W}^{2}\left(1-3 c_{W}^{2}+2 c_{W}^{4}\right) A_{0}\left(\xi M_{W}^{2}\right)-\frac{1}{3} c_{W}^{2} s_{W}^{4} M_{Z}^{2} B_{0}\left(0, \xi M_{W}^{2}, \xi M_{W}^{2}\right)-\left(1-2 c_{W}^{2}\right)\right. \\
& \left.\times\left[B_{22}\left(M_{W}^{2}, M_{h^{0}}^{2}, \xi M_{W}^{2}\right)-B_{22}\left(M_{Z}^{2}, M_{h^{0}}^{2}, \xi M_{Z}^{2}\right)+B_{22}\left(M_{W}^{2}, \xi M_{Z}^{2}, \xi M_{W}^{2}\right)-\left(1-2 c_{W}^{2}\right)^{2} B_{22}\left(M_{Z}^{2}, \xi M_{W}^{2}, \xi M_{W}^{2}\right)\right]\right\} .
\end{aligned}
$$

"Mixed" contributions from diagrams that contain a heavy Higgs boson together with a Goldstone boson or the light $h^{0}$ in the loops do not contribute to either of these two renormalization constants. Such diagrams are suppressed by the factor $\cos (\beta-\alpha)$ and therefore they vanish in the decoupling limit. Purely heavy Higgs-boson contributions to $\delta v$ are of order $\mathcal{O}\left(M_{Z}^{2} / M_{A^{0}}^{2}\right)$ in the $M_{A^{0}} \gg M_{Z}$ limit, and therefore they also vanish. In contrast, for $\delta G^{2}$ we get a nonvanishing contribution,

$$
\delta G^{2 \text { heavy }}=\frac{g^{4}}{96 \pi^{2} c_{W}^{4}}\left(1+2 c_{W}^{4}-2 c_{W}^{2}\right)\left(\Delta_{\epsilon}-\log \frac{M_{A^{0}}^{2}}{\mu_{0}^{2}}\right) .
$$

The only remaining parameters in Eq. (16) still to be fixed are the mass counterterms $\delta m_{1}^{2}, \delta m_{2}^{2}$, and $\delta m_{12}^{2}$. Their expressions are derived from the conditions for $H^{0}$ and $h^{0}$ van- 
ishing renormalized tadpole diagrams and from the on-shell condition for the $A^{0}$ boson. The explicit results for these mass counterterms are given in Eqs. (A4)-(A6) of the Appendix. For completeness, the $H^{0}$ tadpole and the $A^{0}$ selfenergy one-loop results are also presented at the beginning of the Appendix. Then, by implementing all the renormalization constants in Eq. (16), we obtain the vertex function counterterms, separated into light, mixed, and heavy contributions in the $M_{A^{0}} \gg M_{Z}$ limit. The one-point counterterm has already been used for the determination of the basic renormalization constants and is not required for the further discussion; we thus do not list it here:

$$
\begin{aligned}
& \delta \Gamma_{h^{0}}^{(2) \text { light }}=-\frac{g^{2}}{128 \pi^{2} c_{W}^{2}} C_{2 \beta}^{2}\left\{A_{0}\left(M_{h^{0}}^{2}\right)-A_{0}\left(\xi M_{Z}^{2}\right)-\left(2-16 c_{W}^{2}+16 c_{W}^{4}\right) A_{0}\left(\xi M_{W}^{2}\right)+8 B_{22}\left(M_{Z}^{2}, M_{h^{0}}^{2}, \xi M_{Z}^{2}\right)\right. \\
& \left.+8\left(1-2 c_{W}^{2}\right)^{2} B_{22}\left(M_{Z}^{2}, \xi M_{W}^{2}, \xi M_{W}^{2}\right)\right\}, \\
& \delta \Gamma_{h^{0}}^{(3)^{\text {light }}}=\frac{3 g^{3}}{256 \pi^{2} c_{W}^{3}} \frac{1}{M_{Z}} C_{2 \beta}^{2}\left(A_{0}\left(M_{h^{0}}^{2}\right)+A_{0}\left(\xi M_{Z}^{2}\right)+\frac{1}{s_{W}^{2}}\left(2-18 c_{W}^{2}+40 c_{W}^{4}-24 c_{W}^{6}\right) A_{0}\left(\xi M_{W}^{2}\right)\right. \\
& +\frac{4}{3} c_{W}^{2} s_{W}^{2} M_{Z}^{2} B_{0}\left(0, \xi M_{W}^{2}, \xi M_{W}^{2}\right)+4 \frac{1}{s_{W}^{2}}\left(3 c_{W}^{2}-2\right) B_{22}\left(M_{Z}^{2}, M_{h^{0}}^{2}, \xi M_{Z}^{2}\right) \\
& +4\left(1-2 c_{W}^{2}\right) \frac{1}{s_{W}^{2}}\left[B_{22}\left(M_{W}^{2}, M_{h^{0}}^{2}, \xi M_{W}^{2}\right)+B_{22}\left(M_{W}^{2}, \xi M_{Z}^{2}, \xi M_{W}^{2}\right)\right. \\
& \left.\left.+\left(1-2 c_{W}^{2}\right)\left(3 c_{W}^{2}-2\right) B_{22}\left(M_{Z}^{2}, \xi M_{W}^{2}, \xi M_{W}^{2}\right)\right]\right), \\
& \delta \Gamma_{h^{0}}^{(4)}{ }^{\text {light }}=\frac{3 g^{4}}{256 \pi^{2} c_{W}^{4}} \frac{1}{M_{Z}^{2}} C_{2 \beta}^{2}\left(-8 \frac{c_{W}^{2}}{s_{W}^{2}}\left(1-3 c_{W}^{2}+2 c_{W}^{4}\right) A_{0}\left(\xi M_{W}^{2}\right)+\frac{4}{3} c_{W}^{2} s_{W}^{2} M_{Z}^{2} B_{0}\left(0, \xi M_{W}^{2}, \xi M_{W}^{2}\right)\right. \\
& +4\left(1-2 c_{W}^{2}\right) \frac{1}{s_{W}^{2}}\left[B_{22}\left(M_{W}^{2}, M_{h^{0}}^{2}, \xi M_{W}^{2}\right)+B_{22}\left(M_{W}^{2}, \xi M_{Z}^{2}, \xi M_{W}^{2}\right)-B_{22}\left(M_{Z}^{2}, M_{h^{0}}^{2}, \xi M_{Z}^{2}\right)\right. \\
& \left.\left.-\left(1-2 c_{W}^{2}\right)^{2} B_{22}\left(M_{Z}^{2}, \xi M_{W}^{2}, \xi M_{W}^{2}\right)\right]\right), \\
& \delta \Gamma_{h^{0}}^{(2)}=0, \quad \delta \Gamma_{h^{0}}^{(3)^{\text {mixed }}}=0, \quad \delta \Gamma_{h^{0}}^{(4)}=0, \\
& \delta \Gamma_{h^{0}}^{(2)^{\text {heavy }}}=-\frac{g^{2}}{64 \pi^{2} c_{W}^{2}}\left\{M_{A^{0}}^{2}\left(1+2 c_{W}^{2}-3 C_{2 \beta}^{2}\right)\left(\Delta_{\epsilon}+1-\log \frac{M_{A^{0}}^{2}}{\mu_{0}^{2}}\right)\right. \\
& \left.+M_{Z}^{2}\left[9 C_{2 \beta}^{2} S_{2 \beta}^{2}+\frac{1}{6}\left(6-45 C_{2 \beta}^{4}+12 c_{W}^{4}+43 C_{2 \beta}^{2}-14 C_{2 \beta}^{2} c_{W}^{2}+8 C_{2 \beta}^{2} c_{W}^{4}\right)\left(\Delta_{\epsilon}-\log \frac{M_{A^{0}}^{2}}{\mu_{0}^{2}}\right)\right]\right\} \text {, } \\
& \delta \Gamma_{h^{0}}^{(3)^{\text {heavy }}}=-\frac{g^{3}}{64 \pi^{2} c_{W}^{3}} M_{Z} C_{2 \beta}^{2}\left(1+2 c_{W}^{4}-2 c_{W}^{2}\right)\left(\Delta_{\epsilon}-\log \frac{M_{A^{0}}^{2}}{\mu_{0}^{2}}\right), \\
& \delta \Gamma_{h^{0}}^{(4)^{\text {heavy }}}=-\frac{g^{4}}{128 \pi^{2} c_{W}^{4}} C_{2 \beta}^{2}\left(1+2 c_{W}^{4}-2 c_{W}^{2}\right)\left(\Delta_{\epsilon}-\log \frac{M_{A^{0}}^{2}}{\mu_{0}^{2}}\right) .
\end{aligned}
$$


The heavy contributions contain, in addition to the singular $\Delta_{\epsilon}$ part, finite logarithmic heavy mass terms, and for the two-point function quadratic heavy mass terms also.

The renormalized vertex functions in the $M_{A^{0}} \gg M_{Z}$ limit can now be obtained simply by adding the one-loop contributions (9)-(13) and the counterterms (24)-(26). Since it is just an algebraic substitution, we do not present these results explicitly here. However, some comments are in order. First, the quadratic heavy mass terms $\mathcal{O}\left(M_{A^{0}}^{2}\right)$ in the two-point result cancel once we add the one-loop result in Eq. (11) and the counterterm (26). Thus, there are no $\mathcal{O}\left(M_{A^{0}}^{2}\right)$ terms left in any of the renormalized $n$-point functions in the large $M_{A^{0}}$ limit, but still the logarithmic dependence on $M_{A^{0}}$ remains. Second, the renormalized $h_{0}$ Higgs-boson self-energy evaluated at the physical mass $M_{h^{0}}$ allows us to define the MSSM Higgs-boson mass correction $\Delta M_{h^{0}}^{2}$ such that $M_{h^{0}}^{2}=M_{h^{0}}^{2 \text { tree }}$ $+\Delta M_{h^{0}}^{2}$ and $\Delta M_{h^{0}}^{2}=\Delta \Gamma_{R h^{0}}^{(2)}\left(M_{h^{0}}^{2}\right)$. Evaluating the renormalized $h^{0}$ two-point function at $q^{2}=M_{h^{0}}^{2}$, we get the following one-loop mass correction for the light Higgs boson:

$$
\Delta M_{h^{0}}^{2}=\frac{g^{2}}{32 \pi^{2} c_{W}^{2}} M_{Z}^{2}\left(\Psi_{\text {light }}+\Psi_{\text {mixed }}+\Psi_{\text {heavy }}\right),
$$

with

$$
\begin{aligned}
\Psi_{\text {light }}= & \frac{1}{4 M_{Z}^{2}} C_{2 \beta}^{2}\left\{2 A_{0}\left(M_{h^{0}}^{2}\right)+4\left(1-2 c_{W}^{2}\right)^{2} A_{0}\left(\xi M_{W}^{2}\right)+2 A_{0}\left(\xi M_{Z}^{2}\right)-8 B_{22}\left(M_{Z}^{2}, M_{h^{0}}^{2}, \xi M_{Z}^{2}\right)\right. \\
& -8\left(1-2 c_{W}^{2}\right)^{2} B_{22}\left(M_{Z}^{2}, \xi M_{W}^{2}, \xi M_{W}^{2}\right)+M_{Z}^{2} C_{2 \beta}^{2}\left[9 B_{0}\left(M_{h^{0}}^{2}, M_{h^{0}}^{2}, M_{h^{0}}^{2}\right)+B_{0}\left(M_{h^{0}}^{2}, \xi M_{Z}^{2}, \xi M_{Z}^{2}\right)\right. \\
& \left.\left.+2 B_{0}\left(M_{h^{0}}^{2}, \xi M_{W}^{2}, \xi M_{W}^{2}\right)\right]\right\}, \\
\Psi_{\text {mixed }}= & 6 C_{2 \beta}^{2} S_{2 \beta}^{2}\left(\Delta_{\epsilon}+1-\log \frac{M_{A^{0}}^{2}}{\mu_{0}^{2}}\right), \\
\Psi_{\text {heavy }}= & \left(1+3 C_{2 \beta}^{4}+2 c_{W}^{4}-\frac{10}{3} C_{2 \beta}^{2}-\frac{4}{3} C_{2 \beta}^{2} c_{W}^{2}-\frac{2}{3} C_{2 \beta}^{2} c_{W}^{4}\right)\left(\Delta_{\epsilon}-\log \frac{M_{A^{0}}^{2}}{\mu_{0}^{2}}\right) .
\end{aligned}
$$

This mass correction is still UV divergent since we have not included the complete set of diagrams, restricting ourselves to the subset providing contributions that are different from those in the SM. We have checked explicitly that for cancellation of the divergences in the renormalized two-point function it is necessary to include the subset of one-loop diagrams accounting for the gauge-boson contributions. We have also checked that when the gauge-boson contributions are included the $\xi$ gauge dependence in the light one-loop renormalized two-point function disappears. By including all 1PI one-loop contributions, we have checked as well that our results are in agreement with the complete results for the radiative corrections to the Higgs-boson mass listed in the literature [22,23].

On the other hand, the contributions from one-loop diagrams that have at least one heavy Higgs particle ( $\Psi_{\text {mixed }}$ and $\Psi_{\text {heavy }}$ ) contain, in addition to the singular $\Delta_{\epsilon}$ term, some logarithmic heavy mass terms that appear like as nondecoupling effects of the heavy particles at the renormalized Green functions. These apparently nondecoupling effects, however, are not physically observable since they are absorbed into redefinitions of the low energy parameters, more specifically, in the redefinition of the $h^{0}$ mass,

$$
M_{h^{0}}^{2}=M_{h^{0}}^{2^{\text {tree }}}+\Delta M_{h^{0}}^{2}
$$

with $\Delta M_{h^{0}}^{2}$ given in Eq. (27).
By taking into account this MSSM Higgs-boson-mass correction, we can express the renormalized vertex functions, in a generic way, as follows:

$$
\begin{aligned}
& \Gamma_{R h^{0}}^{(2)}=-q^{2}+M_{h^{0}}^{2 \text { tree }}+\Delta M_{h^{0}}^{2}+\Psi_{\mathrm{MSSM}}^{(2) \mathrm{rem}}, \\
& \Gamma_{R h^{0}}^{(3)}=\frac{3 g}{2 M_{Z^{c} c_{W}}}\left(M_{h^{0} \text { tree }}^{2}+\Delta M_{h^{0}}^{2}\right)+\Psi_{\mathrm{MSSM}}^{(3) \mathrm{rem}} \\
& \Gamma_{R h^{0}}^{(4)}=\frac{3 g^{2}}{4 M_{Z^{2}}^{2} c_{W}^{2}}\left(M_{h^{0} \text { tree }}^{2}+\Delta M_{h^{0}}^{2}\right)+\Psi_{\text {MSSM }}^{(4) \mathrm{rem}}
\end{aligned}
$$

where all the singular $\Delta_{\epsilon}$ terms and the logarithmic heavy mass terms are exclusively contained in $\Delta M_{h^{0}}^{2}$. Thus, the apparently nondecoupling terms are absorbed in the redefinition of the $M_{h^{0}}$ Higgs-boson mass. The remaining terms, $\Psi_{\text {MSSM }}^{(2) \text { rem }}, \Psi_{\text {MSSM }}^{(3) \text { rem }}$, and $\Psi_{\text {MSSM }}^{(4) \text { rem }}$ in Eq. (30) come exclusively from the light particle contributions and are finite. For instance, in the two-point function case we have

$$
\Psi_{\mathrm{MSSM}}^{(2) \mathrm{rem}}=\Delta \Gamma_{h^{0}}^{(2)}\left(q^{2}\right)-\Delta \Gamma_{h^{0}}^{(2)}\left(M_{h^{0}}^{2}\right)
$$

with $\Delta \Gamma_{h^{0}}^{(2)}$ given in Eqs. (9) and (11). For the interpretation of the remaining terms it is crucial to have also the corresponding one-loop analysis of the SM self-interactions, which is done in the next section. As a result, it can be 
verified that, in the large $M_{A^{0}} \gg M_{Z}$ limit and by identifying $M_{h^{0}}^{\text {tree }^{2}} \simeq M_{Z}^{2} C_{2 \beta}^{2} \leftrightarrow M_{H_{S M}}^{\text {tree 2 }}$, the remaining terms coincide with the corresponding SM ones.

\section{HIGGS-BOSON SELF-COUPLINGS IN THE SM}

In the standard $\mathrm{SU}(2)_{L} \times \mathrm{U}(1)$ theory, the introduction of one scalar field doublet with nonvanishing vacuum expectation value breaks the gauge symmetry spontaneously to the electromagnetic subgroup U(1). The SM Higgs potential

$$
V(\varphi)=-\mu^{2} \varphi^{\dagger} \varphi+\frac{\lambda}{4}\left(\varphi^{\dagger} \varphi\right)^{2}
$$

contains the complex Higgs doublet $\varphi$ with hypercharge $Y$ $=1$, and the parameters $\lambda$ and $\mu$ related by the vacuum expectation value $\left|\langle\varphi\rangle_{0}\right|^{2}=v^{2} / 2=\mu^{2} / 2 \lambda$.

In order to establish the Higgs mechanism experimentally, the characteristic self-interaction potential of the SM has to be reconstructed once the Higgs particle has been discovered. This task requires the measurement of the selfcouplings of the SM Higgs boson. These self-couplings are uniquely determined by the mass of the Higgs boson, which is related to the quartic coupling $\lambda$ by $M_{H_{\mathrm{SM}}}=\sqrt{2 \lambda} v$. By introducing the physical Higgs field $H=H_{\mathrm{SM}}$ in the neutral component of the doublet, $\varphi^{0}=(v+H) / \sqrt{2}$, the tree-level trilinear and quartic vertices of the Higgs field $H$ can be derived from the potential $V$, yielding

$$
\begin{aligned}
\lambda_{H H H} & =\frac{3 g M_{H_{\mathrm{SM}}}^{2 \text { tree }}}{2 M_{W}}=\frac{3 M_{H_{\mathrm{SM}}}^{2 \text { tree }}}{v}, \\
\lambda_{H H H H} & =\frac{3 g^{2} M_{H_{\mathrm{SM}}}^{2 \text { tree }}}{4 M_{W}^{2}}=\frac{3 M_{H_{\mathrm{SM}}}^{2 \text { tree }}}{v^{2}},
\end{aligned}
$$

with $g$ being the $\mathrm{SU}(2)_{L}$ gauge coupling.

We note once again that the MSSM tree-level selfcouplings (5) reach the corresponding SM couplings above in the decoupling limit. Here we derive the one-loop contributions to the $H_{S M}$ 1PI Green functions, and in particular those that yield the effective triple and quartic self-couplings. Concretely, the generic diagrams from the Higgs sector contributing to the $n$-point SM vertex functions $(n=1, \ldots, 4)$ are shown in Fig. 1 by choosing $\phi \equiv H_{S M}$ and $S$ $\equiv H_{S M}, G^{0}, G^{ \pm}$. The general results for the $n$-point renormalized vertex functions are summarized by the generic expression (6). The tree-level functions for the SM case ( $H$ $\equiv H_{S M}$ ) and for $n=3,4$ correspond to the expressions for the $H_{S M}$ Higgs couplings already given in Eq. (32), and the oneloop contributions are summarized in $\Delta \Gamma_{H_{S M}}^{(n)}$. The computation was done in a general $R_{\xi}$ gauge. The results for $\Delta \Gamma_{H_{S M}}^{(n)}$, in terms of the two-, three-, and four-point one-loop integrals, are given by

$$
\begin{aligned}
\Delta \Gamma_{H_{\mathrm{SM}}}^{(1)}= & \frac{g}{64 \pi^{2} c_{W}} \frac{M_{H_{S M}}^{2}}{M_{Z}}\left\{3 A_{0}\left(M_{H_{S M}}^{2}\right)+A_{0}\left(\xi M_{Z}^{2}\right)+2 A_{0}\left(\xi M_{W}^{2}\right)\right\}, \\
\Delta \Gamma_{H_{\mathrm{SM}}}^{(2)}= & \frac{g^{2}}{128 \pi^{2} c_{W}^{2}} \frac{M_{H_{S M}}^{2}}{M_{Z}^{2}}\left\{3 A_{0}\left(M_{H_{S M}}^{2}\right)+A_{0}\left(\xi M_{Z}^{2}\right)+2 A_{0}\left(\xi M_{W}^{2}\right)+M_{H_{S M}}^{2}\left[9 B_{0}\left(q^{2}, M_{H_{S M}}^{2}, M_{H_{S M}}^{2}\right)\right.\right. \\
& \left.\left.+B_{0}\left(q^{2}, \xi M_{Z}^{2}, \xi M_{Z}^{2}\right)+2 B_{0}\left(q^{2}, \xi M_{W}^{2}, \xi M_{W}^{2}\right)\right]\right\}, \\
\Delta \Gamma_{H_{\mathrm{SM}}}^{(3)}= & \frac{g^{3}}{256 \pi^{2} c_{W}^{3}} \frac{M_{H_{S M}}^{4}}{M_{Z}^{3}}\left\{\left[9 B_{0}\left(q^{2}, M_{H_{S M}}^{2}, M_{H_{S M}}^{2}\right)+B_{0}\left(q^{2}, \xi M_{Z}^{2}, \xi M_{Z}^{2}\right)+2 B_{0}\left(q^{2}, \xi M_{W}^{2}, \xi M_{W}^{2}\right)+(q \rightarrow p)\right.\right. \\
& +(q \rightarrow r)]+2 M_{H_{S M}}^{2}\left[27 C_{0}\left(q^{2}, p^{2}, r^{2}, M_{H_{S M}}^{2}, M_{H_{S M}}^{2}, M_{H_{S M}}^{2}\right)+C_{0}\left(q^{2}, p^{2}, r^{2}, \xi M_{Z}^{2}, \xi M_{Z}^{2}, \xi M_{Z}^{2}\right)\right. \\
& \left.\left.+2 C_{0}\left(q^{2}, p^{2}, r^{2}, \xi M_{W}^{2}, \xi M_{W}^{2}, \xi M_{W}^{2}\right)\right]\right\}, \\
\Delta \Gamma_{H_{\mathrm{SM}}}^{(4)}= & \frac{g^{4}}{512 \pi^{2} c_{W}^{4}} \frac{M_{H_{S M}}^{4}}{M_{Z}^{4}}\left\{\left[9 B_{0}\left((q+p)^{2}, M_{H_{S M}}^{2}, M_{H_{S M}}^{2}\right)+B_{0}\left((q+p)^{2}, \xi M_{Z}^{2}, \xi M_{Z}^{2}\right)+B_{0}\left((q+p)^{2}, \xi M_{W}^{2}, \xi M_{W}^{2}\right)\right.\right. \\
& +(p \rightarrow r)+(p \rightarrow t)]+2 M_{H_{S M}}^{2}\left[27 C_{0}\left(q^{2}, p^{2},(q+p)^{2}, M_{H_{S M}}^{2}, M_{H_{S M}}^{2}, M_{H_{S M}}^{2}\right)\right. \\
& +C_{0}\left(q^{2}, p^{2},(q+p)^{2}, \xi M_{Z}^{2}, \xi M_{Z}^{2}, \xi M_{Z}^{2}\right)+2 C_{0}\left(q^{2}, p^{2},(q+p)^{2}, \xi M_{W}^{2}, \xi M_{W}^{2}, \xi M_{W}^{2}\right) \\
& +(p \rightarrow r)+(p \rightarrow t)+(q \rightarrow t, p \rightarrow r)+(q \rightarrow p, p \rightarrow r)+(q \rightarrow p, p \rightarrow t)] \\
& +2 M_{H_{S M}}^{4}\left[81 D_{0}\left(q^{2}, p^{2}, r^{2}, t^{2},(q+p)^{2},(p+r)^{2}, M_{H_{S M}}^{2}, M_{H_{S M}}^{2}, M_{H_{S M}}^{2}, M_{H_{S M}}^{2}\right)\right. \\
& +D_{0}\left(q^{2}, p^{2}, r^{2}, t^{2},(q+p)^{2},(p+r)^{2}, \xi M_{Z}^{2}, \xi M_{Z}^{2}, \xi M_{Z}^{2}, \xi M_{Z}^{2}\right) \\
& \left.\left.+2 D_{0}\left(q^{2}, p^{2}, r^{2}, t^{2},(q+p)^{2},(p+r)^{2}, \xi M_{W}^{2}, \xi M_{W}^{2}, \xi M_{W}^{2}, \xi M_{W}^{2}\right)+(r \leftrightarrow t)+(p \leftrightarrow r)\right]\right\} . \\
& (33) \\
&
\end{aligned}
$$


These expressions are in general different from the ones obtained in the MSSM [Eq. (9)]. However, they acquire the same structure as in the MSSM in the $M_{A^{0}} \gg M_{Z}$ limit by identifying the light $C P$-even Higgs-boson mass with the SM Higgs mass, that is, $M_{H_{S M}}^{2} \leftrightarrow M_{h^{0}}^{\text {tree }^{2}} \simeq M_{Z}^{2} C_{2 \beta}^{2}$. Consequently, the one-loop light MSSM contributions (9) converge to the SM ones (33) in the decoupling limit. For completeness, we concentrate in the following on the SM vertex counterterms by assuming the on-shell renormalization scheme.

\section{On-shell renormalization in the standard model}

The on-shell renormalization scheme for the SM has been presented in previous articles $[17,20,21]$, to which we refer for details. Here we need only the part for the Higgs sector renormalization. The counterterms are derived from the Higgs potential (31), via multiplicative renormalization,

$$
\begin{aligned}
\varphi & \rightarrow Z_{\varphi}^{1 / 2} \varphi, \\
\lambda & \rightarrow Z_{\lambda} Z_{\varphi}^{-2} \lambda, \quad \mu^{2} \rightarrow\left(\mu^{2}-\delta \mu^{2}\right) Z_{\varphi}^{-1}, \\
v & \rightarrow Z_{\varphi}^{1 / 2}(v-\delta v),
\end{aligned}
$$

and by expanding $Z_{i} \rightarrow 1+\delta Z_{i}$. We obtain the following one-loop counterterms:

$$
\begin{aligned}
\delta \Gamma_{H_{S M}}^{(1)} & =\frac{2 M_{Z} c_{W}}{g} M_{H_{S M}}^{2} \frac{\delta t}{t} \\
\text { with } t & =\frac{2 M_{Z} c_{W} M_{H_{S M}}^{2}}{g}, \\
\delta \Gamma_{H_{S M}}^{(2)} & =\left(q^{2}-M_{H}^{2}\right) \delta Z_{\varphi}-\delta M_{H}^{2}, \\
\delta \Gamma_{H_{S M}}^{(3)} & =-\frac{3 g}{2 M_{Z} c_{W}} M_{H_{S M}}^{2}\left(\delta Z_{\lambda}-\frac{\delta v}{v}\right), \\
\delta \Gamma_{H_{S M}^{(4)}}^{(4)} & =-\frac{3 g^{2}}{4 M_{Z}^{2} c_{W}^{2}} M_{H_{S M}}^{2} \delta Z_{\lambda}
\end{aligned}
$$

with $\delta M_{H}^{2}$ and $\delta t$ related to the original renormalization constants by

$$
\begin{aligned}
\delta M_{H}^{2} & =M_{H}^{2}\left(-3 \frac{\delta v}{v}+\frac{3}{2} \delta Z_{\lambda}-\delta Z_{\varphi}\right)+\delta \mu^{2}, \\
\frac{\delta t}{t} & =\frac{\delta v}{v}-\frac{\delta \mu^{2}}{M_{H_{S M}}^{2}}-\frac{1}{2} \delta Z_{\lambda} .
\end{aligned}
$$

In a first step, the counterterms $\delta t / t$ and $\delta M_{H}^{2}$ are determined from two on-shell conditions in the Higgs sector: the vanishing renormalized tadpole diagram

$$
\Delta \Gamma_{R H_{S M}}^{(1)}=\Delta \Gamma_{H_{S M}}^{(1)}+\delta \Gamma_{H_{S M}}^{(1)}=0,
$$

and the fact that pole of the renormalized Higgs propagator lies at $M_{H_{S M}}^{2}$, which implies

$$
\begin{aligned}
& \Delta \Gamma_{R H_{S M}}^{(2)}\left(M_{H_{S M}}^{2}\right) \\
& \quad=\Delta \Gamma_{H_{S M}}^{(2)}\left(M_{H_{S M}}^{2}\right)+\delta \Gamma_{H_{S M}}^{(2)}\left(M_{H_{S M}}^{2}\right)=0 .
\end{aligned}
$$

Solving these equations, we obtain

$$
\begin{aligned}
\frac{\delta t}{t}= & -\frac{g^{2}}{128 \pi^{2} c_{W}^{2}} \frac{1}{M_{Z}^{2}}\left\{3 A_{0}\left(M_{H_{S M}}^{2}\right)\right. \\
& \left.+A_{0}\left(\xi M_{Z}^{2}\right)+2 A_{0}\left(\xi M_{W}^{2}\right)\right\}, \\
\delta M_{H}^{2}= & \frac{g^{2}}{128 \pi^{2} c_{W}^{2}} \frac{M_{H_{S M}}^{2}}{M_{Z}^{2}}\left\{\left[3 A_{0}\left(M_{H_{S M}}^{2}\right)\right.\right. \\
& \left.+A_{0}\left(\xi M_{Z}^{2}\right)+2 A_{0}\left(\xi M_{W}^{2}\right)\right] \\
& +M_{H_{S M}}^{2}\left[9 B_{0}\left(M_{H_{S M}}^{2}, M_{H_{S M}}^{2}, M_{H_{S M}}^{2}\right)\right. \\
& +B_{0}\left(M_{H_{S M}}^{2}, \xi M_{Z}^{2}, \xi M_{Z}^{2}\right) \\
& \left.\left.+2 B_{0}\left(M_{H_{S M}}^{2}, \xi M_{W}^{2}, \xi M_{W}^{2}\right)\right]\right\} .
\end{aligned}
$$

Next, we need a condition to fix the field-renormalization constant $\delta Z_{\varphi}$. The conventional on-shell condition would be to require unity residue for the physical Higgs-boson propagator, yielding

$$
\begin{aligned}
\delta Z_{\varphi}= & \frac{g^{2}}{128 \pi^{2} c_{W}^{2}} \frac{M_{H_{S M}}^{2}}{M_{Z}^{2}}\left\{9 B_{0}^{\prime}\left(M_{H_{S M}}^{2}, M_{H_{S M}}^{2}, M_{H_{S M}}^{2}\right)\right. \\
& \left.+B_{0}^{\prime}\left(M_{H_{S M}}^{2}, \xi M_{Z}^{2}, \xi M_{Z}^{2}\right)+2 B_{0}^{\prime}\left(M_{H_{S M}}^{2}, \xi M_{W}^{2}, \xi M_{W}^{2}\right)\right\}
\end{aligned}
$$

which is different from zero. For our purpose of comparing the SM and MSSM vertex functions, however, this appears to be inconvenient because in the large $M_{A}$ limit of the MSSM the Higgs field-renormalization constants vanish, as discussed in Sec. II B, and thus the external lines would carry different normalizations in the two models. It is therefore more natural to adopt for the SM a condition that leads to the same normalization and to require

$$
\delta Z_{\varphi}=0
$$

instead of Eq. (40). This is possible because $\delta Z_{\varphi}$ is a UVfinite quantity. With this condition we can compare the two models directly on the basis of the irreducible renormalized vertex functions.

Unlike the previous ones, the $\delta v$ renormalization constant is determined from the gauge sector. We have checked explicitly that the result for $\delta v$ in the SM corresponds to the

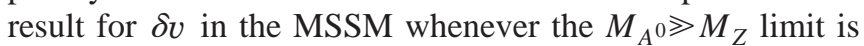
considered and by identifying $M_{h^{0}} \leftrightarrow M_{H_{S M}}$. Thus, the expression for $\delta v / v$ in the SM can be obtained from Eq. (21) by simply replacing $M_{h^{0}}$ by $M_{H_{S M}}$. (36): 


$$
\begin{aligned}
\delta Z_{\lambda}= & \frac{g^{2}}{128 c_{W}^{2} \pi^{2}} \frac{1}{M_{Z}^{2}}\left\{2 A_{0}\left(M_{H_{S M}}^{2}\right)+2 A_{0}\left(\xi M_{Z}^{2}\right)+\frac{4}{s_{W}^{2}}\left(1-c_{W}^{2}-4 c_{W}^{4}+4 c_{W}^{6}\right) A_{0}\left(\xi M_{W}^{2}\right)-\frac{8}{3} c_{W}^{2} s_{W}^{2} M_{Z}^{2} B_{0}\left(0, \xi M_{W}^{2}, \xi M_{W}^{2}\right)\right. \\
& +M_{H_{S M}}^{2}\left[9 B_{0}\left(M_{H_{S M}}^{2}, M_{H_{S M}}^{2}, M_{H_{S M}}^{2}\right)+2 B_{0}\left(M_{H_{S M}}^{2}, \xi M_{W}^{2}, \xi M_{W}^{2}\right)+B_{0}\left(M_{H_{S M}}^{2}, \xi M_{Z}^{2}, \xi M_{Z}^{2}\right)\right] \\
& -8 \frac{c_{W}^{2}}{s_{W}^{2}} B_{22}\left(M_{Z}^{2}, M_{H_{S M}}^{2}, \xi M_{Z}^{2}\right)-\frac{8}{s_{W}^{2}}\left(1-2 c_{W}^{2}\right)\left[B_{22}\left(M_{W}^{2}, M_{H_{S M}}^{2}, \xi M_{W}^{2}\right)+B_{22}\left(M_{W}^{2}, \xi M_{Z}^{2}, \xi M_{W}^{2}\right)\right. \\
& \left.\left.+c_{W}^{2}\left(1-2 c_{W}^{2}\right) B_{22}\left(M_{Z}^{2}, \xi M_{W}^{2}, \xi M_{W}^{2}\right)\right]\right\} .
\end{aligned}
$$

The corresponding vertex counterterms follow immediately via substitution of the SM renormalization constants in Eq. (35). The SM renormalized vertex functions are easily obtained by adding the one-loop contributions (33) and the corresponding counterterms (35)-(42). Remember that the renormalized one-point SM vanishes in the present on-shell renormalization scheme. In addition, the renormalized twopoint SM function also vanishes at the physical mass $M_{H_{\mathrm{SM}}}^{2}$, but not at general $q^{2}$. According to the fact that the renormalized Higgs boson self-energy evaluated at the physical mass squared defines the Higgs-boson mass correction, we obtain trivially that $\Delta M_{H_{S M}}^{2}=0$ (this is nothing other than the on-shell mass condition, which implies $M_{H_{S M}}^{2}$ $\left.=M_{H_{S M}}^{\text {tree2 }}\right)$.

Together with the tree-level SM Higgs self-interactions (32), the renormalized trilinear and quartic $H_{\mathrm{SM}}$ vertex functions at the one-loop level can be written as

$$
\begin{aligned}
& \Gamma_{R H_{S M}}^{(3)}=\frac{3 g}{2 M_{Z} c_{W}} M_{H_{S M}}^{2}+\Psi_{S M}^{(3) \mathrm{rem},}, \\
& \Gamma_{R H_{S M}}^{(4)}=\frac{3 g^{2}}{4 M_{Z}^{2} c_{W}^{2}} M_{H_{S M}}^{2}+\Psi_{S M}^{(4) \mathrm{rem}},
\end{aligned}
$$

where $\Psi_{S M}^{(3)}$ rem and $\Psi_{S M}^{(4)}$ rem are UV-finite functions depending on the external momenta. Remember that similar finite terms were obtained from the light contributions in the MSSM case, summarized in $\Psi_{\text {MSSM }}^{(3) \text { rem }}$ and $\Psi_{\text {MSSM }}^{(4) \text { rem in Eq. }}$ (30). For arbitrary $M_{A^{0}}$ values, these finite contributions are different in the two models. However, for large $M_{A^{0}}$ and by identifying $M_{h^{0}}^{\text {tree }^{2}} \simeq M_{Z}^{2} C_{2 \beta}^{2} \leftrightarrow M_{H_{S M}}^{2}$, we obtain that $\Psi_{M S S M}^{(n) \mathrm{rem}} \rightarrow \Psi_{S M}^{(n) \mathrm{rem}}(n=3,4)$. Thus, these contributions coincide in the $M_{A} 0 \gg M_{Z}$ limit and do not lead to differences between the two models.

\section{DISCUSSION AND CONCLUSIONS}

In order to study whether the SM Higgs sector can be considered as the low-energy effective theory of the MSSM Higgs sector in the $M_{A} 0 \gg M_{Z}$ limit we have compared the predictions in the two theories of the renormalized $n$-point 1PI Green functions for $h^{0}$ and $H_{\mathrm{SM}}$, respectively, at the one-loop level, and for $n=1, \ldots, 4$. We have examined in full detail the veracity of the equality among these functions by comparing them at low-energy scales $p^{2} \ll M_{A^{0}}^{2}$ and by choosing a particular renormalization scheme, the on-shell scheme. This matching [13] between the two theories, via renormalized vertex functions, can be summarized by

$$
\begin{gathered}
\Gamma_{R h^{0}}^{(n) \operatorname{MSSM}_{(}}(p)=\Gamma_{R H_{\mathrm{SM}}}^{(n)} \mathrm{SM}_{(p), \quad p \ll M_{A^{0}}} \\
(n=1, \ldots, 4)
\end{gathered}
$$

where the left-hand side must be understood as the MSSM functions in the $M_{A^{0}} \gg M_{Z}$ limit.

It is worth emphasizing now some important points regarding this comparison of the vertex functions of the two theories. First, as stated in Secs. II and III, the tree-level self-couplings in both models [see Eqs. (5) and (32)] lead to equal results in the SM and in the MSSM vertex functions in the decoupling limit. This implies that the tree-level contributions can be dropped from both sides of the matching conditions (44). Second, as explained in Sec. II, the subset of diagrams that have any number of gauge bosons in the loops gives the same contributions in the SM and in the MSSM (in the $M_{A 0} \gg M_{Z}$ limit) and, therefore, these can also be dropped from both sides of the matching conditions (44). In fact, these kinds of contribution have not been considered explicitly in the present computation. Third, diagrams involving just Goldstone bosons and the lightest Higgs boson in the loops do contribute with nonvanishing corrections which, in principle, are not the same in both models. However, we have demonstrated that the one-loop contributions, given in Eqs. (9) and (33) in the MSSM and the SM, respectively, coincide in the $M_{A^{0}} \gg M_{Z}$ limit. Therefore, they do not contribute either to the differences between the two models in the matching conditions (44). In contrast, we found some light contributions to the vertex counterterms [see Eqs. (24) and (35)-(42)] that are different in both models. These differences in the light sector come from the fact that, whereas the $\delta v / v$ contributions are the same in the SM and in the MSSM in the $M_{A^{0}} \gg M_{Z}$ limit, the other renormalization constants, that is, $\delta Z_{\lambda}$ in the SM and $\delta G^{2}$ in the MSSM, do not coincide. The mass counterterms for the $h^{0}$ and $H_{\mathrm{SM}}$ fields do not coincide either. Thus, what we understand by light 
contributions in this work are also important in the differences between the renormalized vertex functions in both theories.

Overall, we can say that the differences between the oneloop renormalized vertex functions of the two theories in the decoupling limit come, on the one hand, from the one-loop diagrams including at least one heavy MSSM Higgs particle and, on the other hand, from the vertex counterterms. Concretely, Eqs. (10)-(13) give the differences between the oneloop unrenormalized vertex functions of the two theories. Consequently, they cannot be dropped in the conditions (44). Moreover, these different contributions have a finite piece that depends logarithmically and quadratically on the heavy Higgs-boson mass $M_{A^{0}}$ and a divergent piece in $D=4$, and both pieces summarize the potential nondecoupling effects of the heavy Higgs-boson sector of the MSSM. It is essential, however, that these heavy Higgs particle effects can be absorbed into redefinitions of the low-energy parameters, thus not providing any physically observable effect [14]. As we have seen, the counterterms in the SM and in the MSSM are different in both models and therefore they also contribute to the differences between the two models in the matching conditions (44).

Putting all results together and comparing Eqs. (30) and (43), the differences found in the unrenormalized vertex functions are exactly compensated by the $\Delta M_{h^{0}}^{2}$ contribution, and the final results for the renormalized two-, three-, and four-point functions coincide in the two models in the large $M_{A^{0}} \gg M_{Z}$ limit, as required by the matching conditions (44). In other words, all the potential nondecoupling effects from the heavy Higgs modes can be absorbed into the redefinition of the lightest Higgs boson mass $M_{h^{0}}$ [see Eqs. (27) and (30)] and therefore decoupling of the heavy MSSM Higgs particles occurs. We notice that, for arbitrary $M_{A^{0}}$ value, there are other finite terms in the renormalized MSSM $n$-point functions, summarized by the remaining parts $\Psi_{\text {MSSM }}^{(3) \text { rem }}$ and $\Psi_{\text {MSSM }}^{(4) \text { rem }}$ of Eq. (30). However, we have shown that in the SM similar contributions appear in the renormalized $H_{\mathrm{SM}}$ vertex functions, summarized by the remaining parts $\Psi_{\mathrm{SM}}^{(3)}$ rem and $\Psi_{\mathrm{SM}}^{(4) \text { rem }}$ of Eq. (43), which coincide with the corresponding MSSM terms in the large $M_{A^{0}}$ limit. Therefore, these contributions drop out as well in the matching conditions (44).

In conclusion, we have demonstrated that all the apparent nondecoupling one-loop effects from the heavy MSSM Higgs bosons are absorbed in the MSSM Higgs-boson mass $M_{h^{0}}$, and the remaining contributions are suppressed by inverse powers of $M_{A^{0}}$ and therefore vanish in the large $M_{A^{0}}$ limit. Thus, the $h^{0}$ self-interactions converge to the $H_{\mathrm{SM}}$ selfinteractions at the one-loop level and in the $M_{A^{0}} \gg M_{Z}$ limit, and the MSSM $h^{0}$ self-couplings thereby acquire the same structure as the couplings of the SM Higgs boson whenever one identifies $M_{h^{0} \leftrightarrow} M_{H_{S M}}$. Equivalently, we showed that the heavy MSSM Higgs sector decouples from low energy, at the electroweak scale, and leaves behind the SM Higgs sector in the Higgs self-interactions also. Consequently, we would need extremely high-precision experiments for the experimental verification of the SUSY nature of the Higgs boson self-interactions.

\section{ACKNOWLEDGMENTS}

The work of S.P. was supported by the Fundación Ramón Areces. Support by the European Union under HPRN-CT2000-00149 and by the Spanish Ministerio de Ciencia y Tecnología under CICYT projects FPA 2000-0980, FPA 20000956 and PB98-0782 is gratefully acknowledged.

\section{APPENDIX}

In this appendix we display, first, the formulas for the one-loop contributions to the $H^{0}$ tadpole diagram and the $A^{0}$ boson self-energies that are required for on-shell renormalization. Next we present the $\mathcal{O}\left(M_{Z}^{2} / M_{A^{0}}^{2}\right)$ contributions to the renormalization constants $\delta Z_{H_{1,2}}$, which are relevant in order to impose the $A^{0}$-boson on-shell condition. Finally, results for the $\delta m_{1}, \delta m_{2}$, and $\delta m_{12}$ mass counterterms are given. Here we follow the notation introduced throughout this article for light, mixed, and heavy contributions, as explained in Eq. (8).

$H^{0}$ tadpole and $A^{0}$ boson self-energies:

$$
\begin{aligned}
& \Delta \Gamma_{H^{0}}^{(1)}=\frac{g M_{Z}}{64 \pi^{2} c_{W}} C_{2 \beta} S_{2 \beta}\left\{3 A_{0}\left(M_{h^{0}}^{2}\right)+A_{0}\left(\xi M_{Z}^{2}\right)+2 A_{0}\left(\xi M_{W}^{2}\right)\right\}, \\
& \Delta \Gamma_{H^{0}}^{(1)^{\text {heavy }}}=-\frac{g M_{Z}}{32 \pi^{2} c_{W}} C_{2 \beta} S_{2 \beta}\left\{3 M_{A^{0}}^{2}\left(\Delta_{\epsilon}+1-\log \frac{M_{A^{0}}^{2}}{\mu_{0}^{2}}\right)\right. \\
& \left.-\frac{1}{2} M_{Z}^{2}\left[\left(6-12 C_{2 \beta}^{2}+4 c_{W}^{2}\right)+\left(3-9 C_{2 \beta}^{2}+2 c_{W}^{2}\right)\left(\Delta_{\epsilon}-\log \frac{M_{A^{0}}^{2}}{\mu_{0}^{2}}\right)\right]\right\} \text {, } \\
& \Delta \Gamma_{A^{0}}^{(2) \text { light }}\left(M_{A^{0}}^{2}\right)=-\frac{g^{2}}{128 \pi^{2} c_{W}^{2}}\left\{C_{2 \beta}^{2} A_{0}\left(M_{h^{0}}^{2}\right)-\left(2-3 C_{2 \beta}^{2}\right) A_{0}\left(\xi M_{Z}^{2}\right)+2\left(C_{2 \beta}^{2}-2 c_{W}^{2}\right) A_{0}\left(\xi M_{W}^{2}\right)\right. \\
& \left.-2 C_{2 \beta}^{2} S_{2 \beta}^{2} M_{Z}^{2} B_{0}\left(M_{A^{0}}^{2}, M_{h^{0}}^{2}, \xi M_{Z}^{2}\right)\right\},
\end{aligned}
$$




$$
\begin{aligned}
\Delta \Gamma_{A^{0}}^{(2)}{ }^{\text {mixed }}\left(M_{A^{0}}^{2}\right)= & \frac{g^{2}}{64 \pi^{2} c_{W}^{2}} M_{Z}^{2}\left(1+2 c_{W}^{4}+2 C_{2 \beta}^{4}-2 C_{2 \beta}^{2}\right)\left(\Delta_{\epsilon}+2-\log \frac{M_{A^{0}}^{2}}{\mu_{0}^{2}}\right), \\
\Delta \Gamma_{A^{0}}^{(2) \text { heavy }}\left(M_{A^{0}}^{2}\right)= & \frac{g^{2}}{64 \pi^{2} c_{W}^{2}} C_{2 \beta}^{2}\left\{M_{Z}^{2}\left(c_{W}^{2}+\frac{1}{2} S_{2 \beta}^{2}\right)\left(\Delta_{\epsilon}-\log \frac{M_{A^{0}}^{2}}{\mu_{0}^{2}}\right)+3 M_{A^{0}}^{2}\left(\Delta_{\epsilon}+1-\log \frac{M_{A^{0}}^{2}}{\mu_{0}^{2}}\right)\right. \\
& \left.+M_{Z}^{2}\left(1-C_{2 \beta}^{2}\right)\left(1-\frac{\pi}{\sqrt{3}}\right)\right\} .
\end{aligned}
$$

$\delta Z_{H_{1}}$ and $\delta Z_{H_{2}}$ counterterms:

$$
\begin{aligned}
\delta Z_{H_{1}}^{\text {light }} & =-\frac{g^{2}}{64 \pi^{2} c_{W}^{2}} C_{2 \beta} S_{2 \beta}\left[M_{Z}^{2} C_{2 \beta} S_{2 \beta} B_{0}^{\prime}\left(M_{A^{0}}^{2}, M_{h^{0}}^{2}, \xi M_{Z}^{2}\right)+\cot \beta\left(B_{0}+2 B_{1}\right)\left(M_{A^{0}}^{2}, M_{h^{0}}^{2}, \xi M_{Z}^{2}\right)\right], \\
\delta Z_{H_{1}}^{\text {mixed }} & =\frac{g^{2}}{64 \pi^{2} c_{W}^{2}} \frac{M_{Z}^{2}}{M_{A^{0}}^{2}}\left\{\left(1+2 c_{W}^{4}+2 C_{2 \beta}^{4}-2 C_{2 \beta}^{2}\right)-\cot \beta C_{2 \beta} S_{2 \beta}\right\}, \\
\delta Z_{H_{1}}^{\text {heavy }} & =\frac{g^{2}}{64 \pi^{2} c_{W}^{2}} \frac{M_{Z}^{2}}{M_{A^{0}}^{2}}\left\{C_{2 \beta}^{2} S_{2 \beta}^{2}\left(1-\frac{2 \pi}{3 \sqrt{3}}\right)+\cot \beta C_{2 \beta} S_{2 \beta}^{3}\left(2-\frac{\pi}{\sqrt{3}}\right)\right\}, \\
\delta Z_{H_{2}}^{\text {light }} & =-\frac{g^{2}}{64 \pi^{2} c_{W}^{2}} C_{2 \beta} S_{2 \beta}\left[M_{Z}^{2} C_{2 \beta} S_{2 \beta} B_{0}^{\prime}\left(M_{A^{0}}^{2}, M_{h^{0}}^{2}, \xi M_{Z}^{2}\right)-\tan \beta\left(B_{0}+2 B_{1}\right)\left(M_{A^{0}}^{2}, M_{h^{0}}^{2}, \xi M_{Z}^{2}\right)\right], \\
\delta Z_{H_{2}}^{\text {mixed }} & =\frac{g^{2}}{64 \pi^{2} c_{W}^{2}} \frac{M_{Z}^{2}}{M_{A^{0}}^{2}}\left\{\left(1+2 c_{W}^{4}+2 C_{2 \beta}^{4}-2 C_{2 \beta}^{2}\right)+\tan \beta C_{2 \beta} S_{2 \beta}\right\}, \\
\delta Z_{H_{2}}^{\text {heavy }} & =\frac{g^{2}}{64 \pi^{2} c_{W}^{2}} \frac{M_{Z}^{2}}{M_{A^{0}}^{2}}\left\{C_{2 \beta}^{2} S_{2 \beta}^{2}\left(1-\frac{2 \pi}{3 \sqrt{3}}\right)-\tan \beta C_{2 \beta} S_{2 \beta}^{3}\left(2-\frac{\pi}{\sqrt{3}}\right)\right\} .
\end{aligned}
$$

$\delta m_{1}, \delta m_{2}$, and $\delta m_{12}$ counterterms:

$$
\begin{aligned}
\delta m_{1}^{2 \text { light }}= & \frac{g^{2}}{512 \pi^{2} c_{W}^{2}}\left\{16 C_{2 \beta} A_{0}\left(M_{h^{0}}^{2}\right)-4 C_{2 \beta} B_{22}\left(M_{Z}^{2}, M_{h^{0}}^{2}, \xi M_{Z}^{2}\right)\left(5-2 C_{2 \beta}+C_{4 \beta}\right)-2\left(1-2 c_{W}^{2}\right)^{2} B_{22}\right. \\
& \times\left(M_{Z}^{2}, \xi M_{W}^{2}, \xi M_{W}^{2}\right)\left(-2+11 C_{2 \beta}-2 C_{4 \beta}+C_{6 \beta}\right)+2 A_{0}\left(\xi M_{Z}^{2}\right)\left[-2+8 c_{W}^{2}+\left(7-8 c_{W}^{2}\right) C_{2 \beta}-2 C_{4 \beta}+C_{6 \beta}\right] \\
& +A_{0}\left(\xi M_{W}^{2}\right)\left[2+8 c_{W}^{2}-8 c_{W}^{4}+\left(17-44 c_{W}^{2}+44 c_{W}^{4}\right) C_{2 \beta}-2\left(3-4 c_{W}^{2}+4 c_{W}^{4}\right) C_{4 \beta}+3 C_{6 \beta}-4 c_{W}^{2} C_{6 \beta}+4 c_{W}^{4} C_{6 \beta}\right] \\
& \left.+64 M_{Z}^{2} C_{\beta}^{2} C_{2 \beta}^{2} S_{\beta}^{4} B_{0}\left(M_{A^{0}}^{2}, M_{h^{0}}^{2}, M_{Z}^{2}\right)-64 M_{A^{0}}^{2} M_{Z}^{2} C_{2 \beta}^{2} C_{\beta}^{2} S_{\beta}^{4} B_{0}^{\prime}\left(M_{A^{0}}^{2}, M_{h^{0}}^{2}, \xi M_{Z}^{2}\right)\right\} \\
\delta m_{2}^{2 \text { light }}= & \frac{g^{2}}{256 \pi^{2} c_{W}^{2}}\left\{-8 C_{2 \beta} A_{0}\left(M_{h^{0}}^{2}\right)+\left(2+11 C_{2 \beta}+2 C_{4 \beta}+C_{6 \beta}\right) B_{22}\left(M_{Z}^{2}, M_{h^{0}}^{2}, \xi M_{Z}^{2}\right)+\left(1-2 c_{W}^{2}\right)^{2}\left(2+11 C_{2 \beta}\right.\right. \\
& \left.+2 C_{4 \beta}+C_{6 \beta}\right) B_{22}\left(M_{Z}^{2}, \xi M_{W}^{2}, \xi M_{W}^{2}\right)-M_{Z}^{2} C_{\beta}^{2}\left(-1+C_{8 \beta}\right) B_{0}\left(M_{A^{0}}^{2}, M_{h^{0}}^{2}, M_{Z}^{2}\right) \\
& +M_{A^{0}}^{2} M_{Z}^{2} C_{\beta}^{2}\left(C_{8 \beta}-1\right) B_{0}^{\prime}\left(M_{A^{0}}^{2}, M_{h^{0}}^{2}, \xi M_{Z}^{2}\right)+4 A_{0}\left(\xi M_{Z}^{2}\right)\left[-C_{\beta}^{2}\left(2-4 c_{W}^{2}+C_{4 \beta}\right)+S_{\beta}^{2}\right] \\
& \left.-2 A_{0}\left(\xi M_{W}^{2}\right)\left[C_{\beta}^{2}\left(3\left(1-2 c_{W}^{2}\right)^{2}+\left(3-4 c_{W}^{2}+4 c_{W}^{4}\right) C_{4 \beta}\right]-4\left(1-2 c_{W}^{2}+2 c_{W}^{4}\right) S_{\beta}^{2}\right)\right\}, \\
\delta m_{12}^{2 \text { light }}= & -\frac{g^{2}}{128 \pi^{2} c_{W}^{2}} C_{\beta} S_{\beta}\left\{4 C_{2 \beta}^{2} B_{22}\left(M_{Z}^{2}, M_{h^{0}}^{2}, \xi M_{Z}^{2}\right)+4\left(1-2 c_{W}^{2}\right)^{2} C_{2 \beta}^{2} B_{22}\left(M_{Z}^{2}, \xi M_{W}^{2}, \xi M_{W}^{2}\right)-2 A_{0}\left(\xi M_{Z}^{2}\right)\right. \\
& \times\left(1-4 c_{W}^{2}+C_{4 \beta}\right)-A_{0}\left(\xi M_{W}^{2}\right)\left[-1-4 c_{W}^{2}+4 c_{W}^{4}+\left(3-4 c_{W}^{2}+4 c_{W}^{4}\right) C_{4 \beta}\right]+M_{Z}^{2} S_{4 \beta}^{2} B_{0}\left(M_{A^{0}}^{2}, M_{h^{0}}^{2}, M_{Z}^{2}\right) \\
& \left.-M_{A^{0}}^{2} M_{Z}^{2} S_{4 \beta}^{2} B_{0}^{\prime}\left(M_{A^{0}}^{2}, M_{h^{0}}^{2}, \xi M_{Z}^{2}\right)\right\}
\end{aligned}
$$




$$
\begin{aligned}
& \delta m_{1}^{2 \text { mixed }}=\frac{g^{2}}{32 \pi^{2} c_{W}^{2}} M_{Z}^{2} \sin ^{2} \beta\left(1+2 c_{W}^{4}+2 C_{2 \beta}^{4}-2 C_{2 \beta}^{2}\right)\left(\Delta_{\epsilon}+3-\log \frac{M_{A^{0}}^{2}}{\mu_{0}^{2}}\right), \\
& \delta m_{2}^{2 \text { mixed }}=\frac{g^{2}}{32 \pi^{2} c_{W}^{2}} M_{Z}^{2} \cos ^{2} \beta\left(1+2 c_{W}^{4}+2 C_{2 \beta}^{4}-2 C_{2 \beta}^{2}\right)\left(\Delta_{\epsilon}+3-\log \frac{M_{A^{0}}^{2}}{\mu_{0}^{2}}\right) \text {, } \\
& \delta m_{12}^{2 \text { mixed }}=-\frac{g^{2}}{64 \pi^{2} c_{W}^{2}} M_{Z}^{2} S_{2 \beta}\left(1+2 c_{W}^{4}+2 C_{2 \beta}^{4}-2 C_{2 \beta}^{2}\right)\left(\Delta_{\epsilon}+3-\log \frac{M_{A^{0}}^{2}}{\mu_{0}^{2}}\right) . \\
& \delta m_{1}^{2 \text { heavy }}=\frac{g^{2}}{2304 \pi^{2} c_{W}^{2}}\left\{\frac{9}{2} M_{A^{0}}^{2}\left[10+8 c_{W}^{2}-C_{2 \beta}\left(29-8 c_{W}^{2}\right)+6 C_{4 \beta}-3 C_{6 \beta}\right]\left(\Delta_{\epsilon}+1-\log \frac{M_{A^{0}}^{2}}{\mu_{0}^{2}}\right)+C_{2 \beta} S_{2 \beta}^{2} M_{Z}^{2}[9+10 \sqrt{3} \pi\right. \\
& \left.+\left(-2 C_{2 \beta}+C_{4 \beta}\right)(-63+10 \sqrt{3} \pi)\right]+\frac{3}{16} M_{Z}^{2}\left[126-48 c_{W}^{2}+288 c_{W}^{4}-2\left(59-4 c_{W}^{2}+40 c_{W}^{4}\right) C_{2 \beta}\right. \\
& \left.\left.+48 c_{W}^{2}\left(-1+2 c_{W}^{2}\right) C_{4 \beta}-3\left(19-8 c_{W}^{2}+16 c_{W}^{4}\right) C_{6 \beta}-30 C_{8 \beta}+15 C_{10 \beta}\right]\left(\Delta_{\epsilon}-\log \frac{M_{A^{0}}^{2}}{\mu_{0}^{2}}\right)\right\}, \\
& \delta m_{2}^{2 \text { heavy }}=\frac{g^{2}}{2304 \pi^{2} c_{W}^{2}}\left\{\frac{9}{2} M_{A^{0}}^{2}\left[10+8 c_{W}^{2}-C_{2 \beta}\left(8 c_{W}^{2}-29\right)+6 C_{4 \beta}+3 C_{6 \beta}\right]\left(\Delta_{\epsilon}+1-\log \frac{M_{A^{0}}^{2}}{\mu_{0}^{2}}\right)-C_{2 \beta} S_{2 \beta}^{2} M_{Z}^{2}[9+10 \sqrt{3} \pi\right. \\
& \left.+\left(-2 C_{2 \beta}-C_{4 \beta}\right)(63-10 \sqrt{3} \pi)\right]+\frac{3}{16} M_{Z}^{2}\left[126-48 c_{W}^{2}+288 c_{W}^{4}+2\left(59-4 c_{W}^{2}+40 c_{W}^{4}\right) C_{2 \beta}\right. \\
& \left.\left.+48 c_{W}^{2}\left(-1+2 c_{W}^{2}\right) C_{4 \beta}+3\left(19-8 c_{W}^{2}+16 c_{W}^{4}\right) C_{6 \beta}-30 C_{8 \beta}-15 C_{10 \beta}\right]\left(\Delta_{\epsilon}-\log \frac{M_{A^{0}}^{2}}{\mu_{0}^{2}}\right)\right\}, \\
& \delta m_{12}^{2 \text { heavy }}=\frac{g^{2}}{2304 \pi^{2} c_{W}^{2}} S_{2 \beta}\left\{18 M_{A^{0}}^{2}\left(1-3 C_{2 \beta}^{2}+2 c_{W}^{2}\right)\left(\Delta_{\epsilon}+1-\log \frac{M_{A^{0}}^{2}}{\mu_{0}^{2}}\right)-2 C_{2 \beta}^{2} M_{Z}^{2}\left[45-10 \sqrt{3} \pi-C_{2 \beta}^{2}(63-10 \sqrt{3} \pi)\right]\right. \\
& \left.+9 M_{Z}^{2}\left[2+5 C_{2 \beta}^{4}+4 c_{W}^{4}-C_{2 \beta}^{2}\left(5-2 c_{W}^{2}+4 c_{W}^{4}\right)\right]\left(\Delta_{\epsilon}-\log \frac{M_{A^{0}}^{2}}{\mu_{0}^{2}}\right)\right\} .
\end{aligned}
$$

Thereby, $S_{\beta} \equiv \sin \beta, C_{\beta} \equiv \cos \beta, C_{4 \beta} \equiv \cos 4 \beta, C_{6 \beta} \equiv \cos 6 \beta, C_{8 \beta} \equiv \cos 8 \beta$, etc., are used for abbreviations.

[1] M. Carena et al., hep-ph/0010338; D. Cavalli et al., hep-ph/0203056; ECFA/DESY LC Physics Working Group Collaboration, J. A. Aguilar-Saavedra et al., hep-ph/0106315.

[2] J. F. Gunion, H. E. Haber, G. Kane, and S. Dawson, The Higgs Hunter's Guide (Addison-Wesley, Reading, MA, 1990); hep-ph/9302272(E).

[3] H. E. Haber, in "The Decay Properties of SUSY Particles," Proceedings of the 23rd Workshop on the INFN Eloisatron Project, Erice, 1992, pp. 321-372, hep-ph/9305248.

[4] V. Barger, M. S. Berger, A. L. Stange, and R. J. Phillips, Phys. Rev. D 45, 4128 (1992).

[5] P. Osland and P. N. Pandita, Phys. Rev. D 59, 055013 (1999); hep-ph/9911295; hep-ph/9902270.

[6] A. Djouadi, W. Kilian, M. Mühlleitner, and P. M. Zerwas, Eur. Phys. J. C 10, 27 (1999); hep-ph/0001169; M. Mühlleitner, hep-ph/0101262; Ph.D. thesis, Hamburg University, hep-ph/0008127.
[7] A. Djouadi, H. E. Haber, and P. M. Zerwas, Phys. Lett. B 375, 203 (1996).

[8] T. Plehn, M. Spira, and P. M. Zerwas, Nucl. Phys. B479, 46 (1996); B531, 655(E) (1996); R. Lafaye, D. J. Miller, M. Mühlleitner, and S. Moretti, hep-ph/0002238.

[9] W. Hollik and S. Penaranda, Eur. Phys. J. C 23, 163 (2002).

[10] F. Boudjema and A. Semenov, Phys. Rev. D (to be published), hep-ph/0201219.

[11] A. Dobado, M. J. Herrero, and S. Peñaranda, Eur. Phys. J. C 7, 313 (1999); 12, 673 (2000); 17, 487 (2000).

[12] A. Dobado, M. J. Herrero, and S. Peñaranda, in Proceedings of the Workshop on Quantum Effects in the Minimal Supersymmetric Standard Model, Barcelona, 1997, edited by J. Solà (World Scientific, Singapore, 1998), hep-ph/9711441; contribution to the 29th International Conference on High-Energy Physics, Vancouver, 1998, Report No. FTUAM-98-1, hep-ph/9806488. 
[13] H. Georgi, Annu. Rev. Nucl. Part. Sci. 43, 209 (1993); M. J. Herrero and E. Ruiz Morales, Nucl. Phys. B418, 431 (1994); B437, 319 (1995).

[14] T. Appelquist and J. Carazzone, Phys. Rev. D 11, 2856 (1975).

[15] T. Hahn and M. Pérez-Victoria, Comput. Phys. Commun. 118, 153 (1999); T. Hahn, ibid. 140, 418 (2001); T. Hahn and C. Schappacher, ibid. 143, 54 (2002).

[16] G. 't Hooft and M. Veltman, Nucl. Phys. B153, 365 (1979); G. Passarino and M. Veltman, ibid. B160, 151 (1979).

[17] A. Denner, Fortschr. Phys. 41, 307 (1993).

[18] A. Dabelstein, Z. Phys. C 67, 495 (1995); Nucl. Phys. B456, 25 (1995).

[19] P. H. Chankowski et al., Nucl. Phys. B417, 101 (1994); P. Chankowski, S. Pokorski, and J. Rosiek, ibid. B423, 437 (1994).

[20] J. Fleischer and F. Jegerlehner, Phys. Rev. D 23, 2001 (1981).
[21] M. Böhm, H. Spiesberger, and W. Hollik, Fortschr. Phys. 34, 687 (1986); W. Hollik, ibid. 38, 165 (1990).

[22] P. H. Chankowski, S. Pokorski, and J. Rosiek, Phys. Lett. B 274, 191 (1992); A. Brignole, ibid. 281, 284 (1992); D. M. Pierce, J. A. Bagger, K. Matchev, and R. Zhang, Nucl. Phys. B491, 3 (1997).

[23] S. Heinemeyer, W. Hollik, and G. Weiglein, Phys. Lett. B 440, 296 (1998); Phys. Rev. D 58, 091701 (1998); Phys. Lett. B 455, 179 (1999); Acta Phys. Pol. B 30, 1985 (1999); Eur. Phys. J. C 9, 343 (1999); Comput. Phys. Commun. 124, 76 (2000); J. R. Espinosa and R. J. Zhang, J. High Energy Phys. 03, 026 (2000); Nucl. Phys. B586, 3 (2000); M. Carena et al., ibid. B580, 29 (2000); G. Degrassi, P. Slavich, and F. Zwirner, ibid. B611, 403 (2001); A. Brignole, G. Degrassi, P. Slavich, and F. Zwirner, ibid. B631, 195 (2002). 